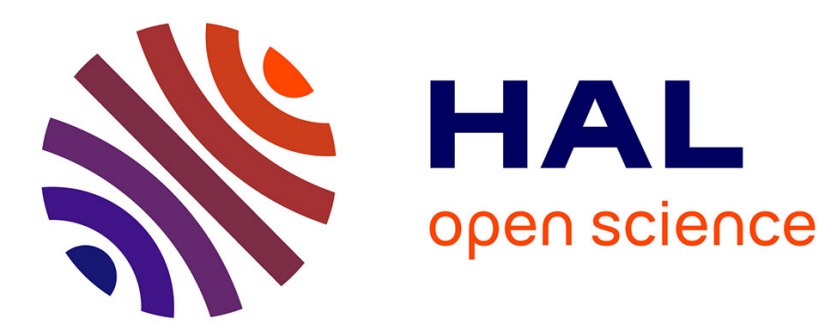

\title{
Solving some Stochastic Partial Differential Equations driven by Lévy Noise using two SDEs. *
}

\author{
Mohamed Mrad
}

\section{To cite this version:}

Mohamed Mrad. Solving some Stochastic Partial Differential Equations driven by Lévy Noise using two SDEs. *. Stochastics: An International Journal of Probability and Stochastic Processes, 2022. hal-03211171

\section{HAL Id: hal-03211171 \\ https://hal.science/hal-03211171}

Submitted on 28 Apr 2021

HAL is a multi-disciplinary open access archive for the deposit and dissemination of scientific research documents, whether they are published or not. The documents may come from teaching and research institutions in France or abroad, or from public or private research centers.
L'archive ouverte pluridisciplinaire HAL, est destinée au dépôt et à la diffusion de documents scientifiques de niveau recherche, publiés ou non, émanant des établissements d'enseignement et de recherche français ou étrangers, des laboratoires publics ou privés. 


\title{
Solving some Stochastic Partial Differential Equations driven by Lévy Noise using two SDEs. *
}

\author{
Mrad Mohamed † \\ mrad@math.univ-paris13.fr
}

April 28, 2021

\begin{abstract}
The method of characteristics is a powerful tool to solve some nonlinear second order stochastic PDEs like those satisfied by a consistent dynamic utilities, see EM13, MM20. In this situation the solution $V(t, z)$ is theoretically of the form $\bar{X}_{t}\left(V\left(0, \bar{\xi}_{t}(z)\right)\right)$ where $\bar{X}$ and $\bar{Y}$ are solutions of a system of two SDEs, $\bar{\xi}$ is the inverse flow of $\bar{Y}$ and $V(0,$.$) is the initial$ condition. Unfortunately this representation is not explicit except in simple cases where $\bar{X}$ and $\bar{Y}$ are solutions of linear equations. The objective of this work is to take advantage of this representation to establish a numerical scheme approximating the solution $V$ using Euler approximations $X^{N}$ and $\xi^{N}$ of $X$ and $\xi$. This allows us to avoid a complicated discretizations in time and space of the SPDE for which it seems really difficult to obtain error estimates. We place ourselves in the framework of SDEs driven by Lévy noise and we establish at first a strong convergence result, in $\mathbf{L}_{p}$-norms, of the compound approximation $X_{t}^{N}\left(Y_{t}^{N}(z)\right)$ to the compound variable $X_{t}\left(Y_{t}(z)\right)$, in terms of the approximations of $X$ and $Y$ which are solutions of two SDEs with jumps. We then apply this result to Utility-SPDEs of HJB type after inverting monotonic stochastic flows.
\end{abstract}

\section{Introduction}

Solving PDEs using the characteristics method is powerful tool in the theory of differential equations. This method has been extended to the framework of stochastic PDEs in chapter 6 of Kunita's book [Kun97] and called the stochastic characteristics method. It consists in studying the solution $U(t, z)$ of the equation along the path of a process $Y_{t}(z)$. If $Y$ is well chosen, the process $X_{t}(z):=U\left(t, Y_{t}(z)\right)$ should satisfy a differential equation of lower order and much simpler to study. This method works very well since the $Y$ process is such that $y \mapsto Y_{t}(y)$ is homeomorphic flow. In this case, by making a variable change, the solution of our initial problem is written $U(t, z)=X_{t}\left(Y_{t}^{-1}(z)\right)$. Unfortunately, apart from a few special cases, it is complicated, if not impossible, to obtain closed formulas for the characteristics $X$ and $Y$ (or its inverse $Y^{-1}$ ). Let $X^{N}$ be an approximation of $X$ and $\xi^{N}$ be an approximation of $\xi:=Y^{-1}$ for some convergence parameter $N \rightarrow+\infty$, our aim is to show that the compound approximation $X^{N}\left(\omega, \xi^{N}(\omega)\right)$ converges to $X(\omega, \xi(\omega))$. For this we need to answer the following questions: Which scheme to approximate the inverse flow $\xi$ ? Under which assumptions does the compound approximation $\omega \mapsto$ $X^{N}\left(\omega, \xi^{N}(\omega)\right)$ converge to the compound map $\omega \mapsto X(\omega, \xi(\omega))$ and in which manner? And

\footnotetext{
*strong approximation, Euler scheme, stochastic flow, method of stochastic characteristics, SPDE driven by Lévy noise, Utility-SPDE, Garsia-Rodemich-Rumsey lemma

${ }^{\dagger}$ Laboratoire Analyse, Géométrie et Applications (LAGA, UMR CNRS 7539), Université Paris 13
} 
finally, what is the convergence rate and how does it depend on those related to the approximations $X^{N}$ to $X$ and $\xi^{N}$ to $\xi$ ?

It is easy to see that our study becomes simple if we make the hypothesis that the pair $\left(X, X^{N}\right)$ is independent of $\left(\xi, \xi^{N}\right)$, by using a conditioning argument. This is not the case in this work as we will explain later. The study is therefore more complex and the classic arguments are not necessarily effective.

As the strong convergence rates constitute the corner stone for designing efficient Multi-Level Monte Carlo methods which significantly speed-up crude Monte Carlo methods by reducing the variance [Hei01, Gil08] or can remove the simulation bias where the number of levels is randomized [RG12, GM16], our aim is to study the strong convergence (convergence in $\mathbf{L}_{p^{-}}$ norms) in this setting.

Fortunately, a first work with E. Gobet [GM18] answers some of these questions in a very general framework and establish a strong convergence rates for compound maps in complicated situations where the error analysis was not available so far. Theorem 1 in GM18 gives a strong approximation of the compound $F_{t}\left(\Theta_{t}(\theta)\right)$ where the random fields $F$ and $\Theta$ are arbitrary (not a necessarily a semimartingales) and satisfy some space regularities assumptions. The scope of application of this result is potentially large: the application to utility-SPDE without jumps is developed in Section 3 of GM18 and applications to stochastic processes (possibly non semimartingales) at random times (possibly non stopping times) are considered in [GM16], where the nice interplay with unbiased simulation scheme of Rhee-Glynn [RG12] is presented.

The starting idea of this work and that of [GM18] comes essentially from a joint works with N. El Karoui [EM13] and with A. Matoussi [MM20] on the field of stochastic consistent utilities, introduced under the name of forward utilities by M. Musiela and T. Zariphopoulou [MZ07. This new concept which generalizes the classic utility functions, models possible changes over the time of both the individual preferences of an agent and the dynamic of the universe of investment. This works always leads us to completely non-linear stochastic PDEs. The originality is that we have succeeded in linking the solutions of these SPDEs with those of two SDEs. We have shown that the marginal utility is represented as the compound of two SDE's solutions. This explains the need to study the composition of the approximations of the two SDEs instead of trying to directly discretize the SPDEs, which is not an easy task. To be clearer, a dynamic utility of an investor is a random maps $(t, z, \omega) \mapsto U(t, z, \omega) \in \mathbb{R}$, depending on time $t$ and on the wealth $z \in \mathbb{R}$. Assume it is of Itô type driven by a Lévy process, i.e.

$$
\mathrm{d} U(t, z)=\beta(t, z) \mathrm{d} t+\gamma(t, z) \cdot \mathrm{d} W_{t}+\int_{\mathbb{R}} H(t, z, e) \tilde{N}(d t, d e),
$$

where $W$ is a $d$-dimensional Brownian motion and $\tilde{N}$ the compensated version of an independent Lévy random measure $N$ on $[0, \infty) \times \mathbb{R}$ with intensity measure $\lambda(t) d t \times \nu(d e)$. The triplet $(\beta, \gamma, H)$ denotes the local characteristics of $U$ with values in $\mathbb{R} \times \mathbb{R}^{d} \times \mathbb{R}$. Denote by $U_{z}$ and $U_{z z}$ the first and the second derivative of $U$ with respect to $z$. Then, the dynamic utility $U$, associated to the investor's portfolio optimization, is consistent if it solves the second-order fully nonlinear SPDE driven by Lévy noise and of HJB type [MM20] (the drift $\beta$ is necessarily constrained)

$$
\begin{aligned}
d U(t, z) & =\left(-z U_{z}(t, z) r_{t}+\int_{\mathbb{R}}(U(t, z)+H(t, z, e)) \nu(d e)-\mathcal{Q}\left(t, z, \kappa^{*}\right)\right) d t \\
& +\gamma(t, z) d W_{t}+\int_{\mathbb{R}} H(t, z, e) \tilde{N}(d t, d e) .
\end{aligned}
$$

Where,

$$
\mathcal{Q}\left(t, z, \kappa^{*}\right)=\int_{\mathbb{R}}(U+H)\left(t, z\left(1+\kappa^{*}(t, z) h^{S}(t, e)\right)\right) \nu(d e)-\frac{1}{2 U_{z z}}\left\|\gamma_{z}^{\mathcal{R}}+U_{z}(t, z)\left(\eta_{t}-\alpha_{t}\right)\right\|^{2}
$$




$$
+\frac{1}{2 U_{z z}(t, z)}\left\|\int_{\mathbb{R}}\left(U_{z}+H_{z}\right)\left(t, z\left(1+\kappa^{*}(t, z) h^{S}(t, e)\right)\right) \cdot h^{S}(t, e) \nu(d e)\right\|^{2} .
$$

With $r$ and $\eta$ denote the interest rate and the risk premium of the market. The quantity $z \kappa^{*}(t, z)$ denotes the strategy of the investor and is given by

$z \kappa^{*}(t, z)=-\frac{\gamma_{z}^{\mathcal{R}}(t, z)+U_{z}(t, z)\left(\eta_{t}-\alpha_{t}\right)}{U_{z z}(t, z)}-\frac{\int_{\mathbb{R}}\left(U_{z}+H_{z}\right)\left(t, z\left(1+\kappa^{*}(t, z) h^{S}(t, e)\right)\right) \cdot h^{S}(t, e) \nu(d e)}{U_{z z}(t, z)}$.

In the continuous case ([EM13, Section 3]), this equation reduces, to

$$
\mathrm{d} U(t, z, \omega)=\left(-r_{t} z U_{z}+\frac{1}{2 U_{z z}}\left\|\gamma_{z}+U_{z} \eta_{t}\right\|^{2}\right)(t, z, \omega) \mathrm{d} t+\gamma(t, z, \omega) \cdot \mathrm{d} W_{t} .
$$

Moreover, see [EM13, Section 4] for equation (2) and [MM20, Theorem 3.8] for equation (1), we represent the spatial first derivative of the SPDE solution as the compound of the solutions of two SDEs with explicit coefficients and driven by the same Brownian motion $W$ and the same Lévy random measure $N$; denote them by $\left(X_{t}(x): t \geq 0\right)$ and $\left(Y_{t}(y): t \geq 0\right)$, parameterized by their initial space conditions $x$ and $y$ at time 0 . More precisely, the utility-SPDE (2) admits a unique concave (with respect to the space variable $z$ ) solution with marginal $U_{z}$ characterized by composition of stochastic flows:

$$
U_{z}(t, z, \omega)=X_{t}\left(u_{z}\left(0, \xi_{t}(z, \omega)\right), \omega\right), \quad U(0, z, \omega)=u(0, z)
$$

where $\xi_{t}(z)$ : denotes the inverse flow of $y \mapsto Y_{t}(y)$. This connection between utility-SPDE and SDEs allows to solve theoretically the problem but the numerical resolution remains an open issue. In particular we would like, as mentioned above, to avoid a complicate discretization in time and space of the SPDE (1) and we would like to take advantage of the representation (3) as compound random maps. Briefly, the question can be formulated as follows: let $N$ be a number of discretization times (including jump times) and assume that an approximation $\xi^{N}$ converge to $\xi$ as $N \rightarrow+\infty$ with order $\alpha^{\xi}$, and $X^{N}$ to $X$ with order $\alpha^{X}$, both convergences being considered in the $L_{p}$ sense.

- Does $X_{t}^{N}\left(u_{z}\left(0, \xi_{t}^{N}(z)\right)\right)$ converges to $X_{t}\left(u_{z}\left(0, \xi_{t}(z)\right)\right.$ as $N \rightarrow+\infty$ ?

- What is the convergence rate, and how it depends on $\alpha^{X}$ and $\alpha^{\xi}$ ?

Note that, in the case of continuous semimartingale, we have addressed these issues in GM18, Theorem 8] where we have shown, using Euler's schemes to approximate $X$ and $\xi$, that $X_{t}^{N}\left(u_{z}\left(0, \xi_{t}^{N}(z)\right)\right)$ converge with order $\frac{1}{2}$ to the first derivative $U_{z}(t, z)$ of the solution $U$ of (2) satisfying $U(t, z)=u(z)$. The purpose of this paper is to establish an equivalent result for more complex family of SPDE's driven by a Lévy noise and in particular for the utility-SPDE (1).

The paper is organized as follows. In Section 2, we recall a general convergence result GM18, Theorem 1] estimating the $\mathbf{L}_{p}$-error $\left\|F^{N}\left(\Theta^{N}\right)-F(\Theta)\right\|_{\mathbf{L}_{p}}$ by assuming locally uniform approximations of $F^{N}-F$, and local-Hölder continuity on $F$. In Section 3 , we study the error induced by compound Euler schemes related to SDEs with jumps, through their initial conditions. The major difficulty is to show that the hypotheses of [GM18, Theorem 1] are satisfied in order to be able to apply this result to the framework of this paper. Several intermediate results, whose proofs are long and complex, are necessary to verify all these assumptions and establish our main result Theorem 5 which show that the convergence rate for the approximation of compound SDEs (strongly dependent because built with the same Brownian motion and the same Lévy measure) inherits from that of simple Euler scheme for each SDE. In Section 4 , we come back to the application to utility-SPDE (1). 


\section{$2 \quad \mathrm{~L}_{p}$-approximation of compound random maps: recent results}

Let $(\mathcal{E},||$.$) be a separable Banach space and (\Omega, \mathcal{F}, \mathbb{P})$ be a probability space. Let $F$ be a random field, i.e. a $\mathcal{F} \otimes \mathcal{B}\left(\mathbb{R}^{d}\right)$-measurable mapping $(\omega, x) \in\left(\Omega, \mathbb{R}^{d}\right) \mapsto F(\omega, x) \in \mathcal{E}$, continuous in $x$ for a.e. $\omega$ and let a $\mathbb{R}^{d}$-valued $\mathcal{F}$-random variable $\Theta: \Omega \mapsto \mathbb{R}^{d}$. Denote by $F^{N}$ and $\Theta^{N}$ the approximations of $F$ and $\Theta$, where $N \rightarrow+\infty$ is a asymptotic parameter.

For $p>0$ and for a random variable $Z: \Omega \mapsto \mathcal{E}$ or $\mathbb{R}^{d}$, we set $\|Z\|_{\mathbf{L}_{p}}=\left(\mathbb{E}|Z|^{p}\right)^{1 / p}$ and say that $Z \in \mathbf{L}_{p}$ if $\|Z\|_{\mathbf{L}_{p}}<+\infty$. Despite $\|\cdot\|_{\mathbf{L}_{p}}$ not being a norm for $p<1$, we refer to it as $\mathbf{L}_{p}$-norm to simplify the discussion.

Let us now give the assumptions under which a general convergence result for the compound $F^{N}\left(\Theta^{N}\right)$ to $F(\Theta)$ has been established in [GM18]. They ensure that all the quantities of interest to us belong to any $\mathbf{L}_{p}$, with some locally uniform estimates w.r.t. the space dependence.

(H1) For any $p>0$, there exist constants $\alpha_{p}^{\sqrt{\mathbf{H 1} 1}} \in[0,+\infty)$ and $C_{p}^{\sqrt{\mathbf{H 1} 1}} \in[0,+\infty)$ such that

$$
\left\|\sup _{|x| \leq \lambda}|F(\cdot, x)|\right\|_{\mathbf{L}_{p}} \leq C_{p}^{\sqrt{\mathbf{H 1} 1}} \lambda^{\alpha \stackrel{\text { H1 }}{~}}, \quad \forall \lambda \geq 1 .
$$

(H2) There is a $\kappa \in(0,1]$ such that for any $p>0$, there exist constants $\alpha_{p}^{\sqrt{\mathbf{H 2} 2}} \in[0,+\infty)$ and $C_{p}^{\overline{\mathbf{H 2} 2}} \in[0,+\infty)$ such that

$$
\left\|\sup _{x \neq y,|x| \leq \lambda,|y| \leq \lambda} \frac{|F(\cdot, y)-F(\cdot, x)|}{|y-x|^{\kappa}}\right\|_{\mathbf{L}_{p}} \leq C_{p}^{\sqrt{\mathbf{H 2} 2}} \lambda^{\alpha \frac{\sqrt{\mathbf{H 2} 2}}{p}}, \quad \forall \lambda \geq 1 .
$$

(H3) For any $p>0$, there exist a constant $\alpha_{p}^{[\mathbf{H 3}]} \in[0,+\infty)$ and a sequence $\left(\varepsilon_{p}^{N,[\mathbf{H 3}]}\right)_{N \geq 1}$ with $\varepsilon_{p}^{N, \mathrm{H3}]} \in[0,+\infty)$ such that

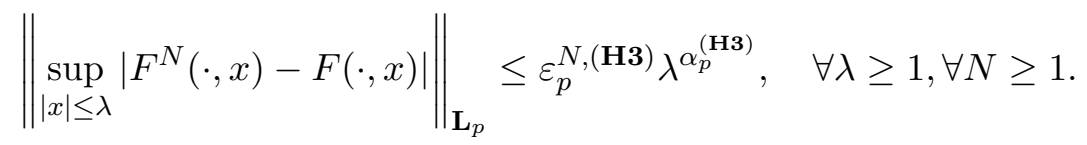

(H4) For any $p>0$, there exist a constant $C_{p}^{\sqrt{\mathbf{H 4}-\mathrm{a} a}} \in[0,+\infty)$ and a sequence $\left(\varepsilon_{p}^{N, \overline{\mathbf{H 4}-\mathrm{b} b}}\right)_{N \geq 1}$ with $\varepsilon_{p}^{N, \underline{H} 4-\mathrm{b}]} \in[0,+\infty)$ such that

$$
\begin{aligned}
\|\Theta\|_{\mathbf{L}_{p}} \vee\left\|\Theta^{N}\right\|_{\mathbf{L}_{p}} \leq C_{p}^{\text {H4-a }}, \quad \forall N \geq 1, \\
\left\|\Theta^{N}-\Theta\right\|_{\mathbf{L}_{p}} \leq \varepsilon_{p}^{N, \text { H4-b }}, \quad \forall N \geq 1 .
\end{aligned}
$$

Under these assumptions, the following Theorem states an error estimate on the approximation of $F(\Theta)$ by $F^{N}\left(\Theta^{N}\right)$, as a function of $N$, through the sequences $\left(\varepsilon^{N, \overline{\mathbf{H 3}}}\right)_{N \geq 1}$ and $\left(\varepsilon^{N, \overline{\mathbf{H} 4-\mathrm{b}}}\right)_{N \geq 1}$.

Theorem 1 (Gobet-Mrad [GM18]). Assume $(\mathbf{H 1})-(\mathbf{H 2})-(\mathbf{H 3})-(\mathbf{H} 4-\mathrm{a})-(\mathbf{H} 4-\mathrm{b})$. Then for any $p>0$ and any $p_{2}>p$, there is a constant $c_{4}$ independent on $N$ such that

$$
\left\|F^{N}\left(\Theta^{N}\right)-F(\Theta)\right\|_{\mathbf{L}_{p}} \leq c_{\llbracket}\left(\varepsilon_{2 p}^{N, \overline{\mathbf{H 3} 3}}+\left[\varepsilon_{\kappa p 2}^{N, \overline{\mathbf{H 4}-b}]}\right]^{\kappa}\right), \quad \forall N \geq 1 .
$$

Quite intuitively, the global approximation error inherits the rates from that on $F$ and that on $\Theta$ modified by the local Hölder regularity of $x \mapsto F(\omega, x)$. 
Remark 2.1. In its original version, this result is given in two variants, the first is the one recalled here and the second one gives an equivalent conclusion but instead of the $\lambda$-polynomial dependency of the upper bounds in (H1-H2-H3), we consider exponential dependency. This allow us to analyse the approximation of diffusion process in diffusion time $Z_{t}=X_{\left|Y_{t}\right|}$ see [AZ01] and [GM16] for this and other applications of this second variant.

Remarks and comments This is a very general result but the assumptions necessary to apply it and especially (H1-H2-H3) are sometimes very complicated to verify. For example, if $x$ is the time variable, one can verify that (H1-H2-H3) are satisfied by using Doob inequalities or other martingale estimates. But in other situations this verification can be complex. One can think of using the Kolmogorov continuity criterion for random fields [Kun97, Theorem 1.4.1 p.31], but it does not yield the quantitative estimates we are looking for. However, there is an interesting result that gives refinement compared to the Kolmogorov criterion. It is about the Garsia-Rodemich-Rumsey lemma [GRR70] (see [Nua06, p.353-354]). This lemma allows us to go from pointwise estimates to locally uniform estimates, by assuming Hölder regularity in $\mathbf{L}_{p}$. It has been very useful to us in studying SPDEs in a continuous setting and will undoubtedly be useful in this work as well. In the literature, this approach has been extensively developed in BY82 for studying regularity of local times of continuous martingales w.r.t. the space variable.

The following two results will be used frequently in this paper. The first key result is obtained from Garsia-Rodemich-Rumsey lemma [GRR70], see the proof of [GM18, Theorem 2].

Theorem 2 (Gobet-Mrad [GM18]). Let $p>d$. Assume that $G$ is $\mathcal{F} \otimes \mathcal{B}\left(\mathbb{R}^{d}\right)$-measurable mapping $(\omega, x) \in\left(\Omega, \mathbb{R}^{d}\right) \mapsto G(\omega, x) \in \mathcal{E}$, continuous in $x$ for a.e. $\omega$. Assume that $G(x)$ is in $\mathbf{L}_{p}$ for any $x$ and that there exist constants $C^{(G)} \in[0,+\infty), \beta^{(G)} \in(d / p, 1]$ and $\tau^{(G)} \in[0,+\infty)$ such that

$$
\|G(x)-G(y)\|_{\mathbf{L}_{p}} \leq C^{(G)}|x-y|^{\beta^{(G)}}(1+|x|+|y|)^{\tau^{(G)}}, \quad \forall(x, y) \in \mathbb{R}^{d} \times \mathbb{R}^{d} .
$$

Then, for any $\beta \in\left(0, \beta^{(G)}-d / p\right)$, we have

$$
\left\|\sup _{x \neq y,|x| \leq \lambda,|y| \leq \lambda} \frac{|G(y)-G(x)|}{|y-x|^{\beta}}\right\|_{\mathbf{L}_{p}} \leq c_{6} C^{(G)} \lambda^{\tau^{(G)}+\beta^{(G)}-\beta}, \quad \forall \lambda \geq 1,
$$

where $c_{[6]}$ is a constant depending only on $d, p, \beta, \beta^{(G)}, \tau^{(G)}$.

A similar result is proved in [RY99, Theorem 2.1, p.26] using the Kolmogorov criterion, with $x$ and $y$ in a compact set, i.e. with $\tau^{(G)}=0$; the quoted result is not sufficient for our study.

As a consequence, we obtain the following result that may serve to easily check $(\mathbf{H 1})$.

Corollary 1. Let consider the assumptions and notations of Theorem 2. Then we have

$$
\left\|\sup _{|x| \leq \lambda}|G(x)|\right\|_{\mathbf{L}_{p}} \leq c_{77} \lambda^{\tau^{(G)}+\beta^{(G)}}, \quad \forall \lambda \geq 1,
$$

where $c_{[7]}:=\|G(0)\|_{\mathbf{L}_{p}}+c_{[6} C^{(G)}$ where $c_{[6}$ is defined in Theorem 2 with $\beta=\left(\beta^{(G)}-d / p\right) / 2$. In particular, the constant $c_{6 \sqrt{6}}$ depends only on $d, p, \beta^{(G)}, \tau^{(G)}$. 


\section{Application to compound Euler schemes}

\subsection{Problem's setting}

In this section, we fix finite time horizon $T$ and we consider a standard filtered probability space $(\Omega, \mathbb{F}, \mathbb{P})$ supporting two $q$-dimensional standard Brownian motions $W=\left(W^{1}, \ldots, W^{q}\right)$ and $B=\left(B^{1}, \ldots, B^{q}\right)$ on $[0, T]$ and an independent Lévy random measure $N$ on $[0, \infty) \times \mathbb{R}^{q^{\prime}}$ with deterministic time dependent intensity measure $\lambda(t) d t \times \nu(d e)$ defined on the probability space $(\Omega, \mathcal{F}, \mathbb{F}, \mathbb{P}) . \quad \lambda$ is the time intensity of jumps with $\lambda([0, T])=\left[0, \lambda_{\max }\right]$ for some $\left.\lambda_{\max } \in\right] 0, \infty[$ and $\nu$ is a positive measure on $\mathbb{R}^{q^{\prime}}$ with finite intensity, i.e., $\nu\left(\mathbb{R}^{q^{\prime}}\right)=\int_{\mathbb{R}^{q^{\prime}}} \nu(d e)<\infty$. We also denote by $\tilde{N}$ the compensated version of $N$ :

$$
\tilde{N}(d t, d e)=N(d t, d e)-\nu(d e) \lambda(t) d t .
$$

We are concerned by two $\mathbb{R}^{d}$-valued stochastic processes $X$ and $Y$, solutions of the following stochastic differential equations (SDE for short)

$$
\begin{aligned}
\mathrm{d} X_{t}(x) & =\mu\left(t, X_{t}(x)\right) \mathrm{d} t+\sum_{i=1}^{q} \sigma_{i}\left(t, X_{t}(x)\right) \mathrm{d} W_{t}^{i}+\int_{\mathbb{R}^{q^{\prime}}} h\left(t, X_{t^{-}}(y), e\right) \tilde{N}(d t, d e), \quad X_{0}(x)=x, \\
\mathrm{~d} Y_{t}(y) & =b\left(t, Y_{t}(y)\right) \mathrm{d} t+\sum_{i=1}^{q} \gamma_{i}\left(t, Y_{t}(y)\right) \mathrm{d} B_{t}^{i}+\int_{\mathbb{R}^{q^{\prime}}} g\left(t, Y_{t^{-}}(y), e\right) \tilde{N}(d t, d e), \quad Y_{0}(y)=y,
\end{aligned}
$$

where $\mu, b, \sigma_{i}, \gamma_{i}$ are deterministic functions from $[0, T] \times \mathbb{R}^{d}$ into $\mathbb{R}^{d}$ and $h, g$ are deterministic functions from $[0, T] \times \mathbb{R}^{d} \times \mathbb{R}^{q^{\prime}}$ into $\mathbb{R}^{d \times q^{\prime}}$, globally Lipschitz in space to ensure the existence of a unique strong solution. Depending on the potential applications, we may require that $B$ and $W$ are the same, or different, or built from each other, possibly in complicate ways. Actually, observe that we do not assume that the couple $(B, W)$ forms a higher-dimensional Brownian motion: this general setting allows flexibility in further applications. As an example for solving utility-SPDE in Section 4, we need to consider $B$ as the backward Brownian motion of $W$.

Denote by $X_{T}^{N}(x)\left(\operatorname{resp} . Y_{T}^{N}(y)\right)$ the Euler scheme with time step $T / N$ of $X_{T}(x)\left(\operatorname{resp} . Y_{T}(y)\right)$ : Using previous results, we aim at establishing a new convergence result of the compound scheme $X_{t}^{N}\left(Y_{t}^{N}(y)\right)$ to the compound SDE $X_{t}\left(Y_{t}(y)\right)$ as $N$ goes to infinity. More precisely, we prove in the following, under precise assumptions, see Theorem 5 , that

$$
\left\|X_{t}^{N}\left(Y_{t}^{N}(y)\right)-X_{t}\left(Y_{t}(y)\right)\right\|_{\mathbf{L}_{p}}=O\left(N^{-1 / 2}\right), \forall y \in \mathbb{R}^{d}, p>0 .
$$

As explained in the introduction, this approximation issue is actually motivated by the resolution of some SPDEs (with or without jumps) by composition of stochastic flows. Relating compound SDEs to SPDEs is, in a sense, obvious since it is sufficient to apply an extension of the Itô-Ventzel's formula established by B. Øksendal and T. Zhang [OZ07][Theorem 3.1] to the compound process $V(t, y):=X_{t}\left(Y_{t}(y)\right)$. Under good regularity assumptions on $(\mu, \sigma, h)$, the quoted result shows that $V(t, y):=X_{t}\left(Y_{t}(y)\right)$ is still a semimartingale, solution of a second order SPDE, with stochastic coefficients, given by

$$
\begin{gathered}
d V(t, y)=\left(\partial_{y} V(t, y) \frac{b\left(t, Y_{t}(y)\right)}{\partial_{y} Y_{t}(y)}+\frac{1}{2}\left(\partial_{y}^{2} V(t, y)-\partial_{y} V(t, y) \frac{\partial_{y}^{2} Y_{t}(y)}{\partial_{y} Y_{t}(y)}\right) \frac{\gamma^{2}\left(t, Y_{t}(y)\right)}{\left(\partial_{y} Y_{t}(y)\right)^{2}}\right. \\
\left.+\mu(t, V(t, y))+\partial_{y} V(t, y)\left(\partial_{x} \sigma\right)(t, V(t, y)) \frac{\gamma\left(t, Y_{t}(y)\right)}{\partial_{y} Y_{t}(y)}\right) \mathrm{d} t \\
+\left(\partial_{y} V(t, y) \frac{\gamma\left(t, Y_{t}(y)\right)}{\partial_{y} Y_{t}(y)}+\sigma(t, V(t, y))\right) \mathrm{d} W_{t}
\end{gathered}
$$




$$
\begin{gathered}
+\int_{\mathbb{R}}\left(\bar{V}(t, y, e)-V(t, y)-h(t, V(t, y), e)-\frac{\partial_{y} V(t, y)}{\partial_{y} Y_{t}(y)} g\left(t, Y_{t}(y), e\right) \lambda(t) \nu(d e) d t\right. \\
+\int_{\mathbb{R}}\left(\bar{V}(t, y, e)-V\left(t^{-}, y\right)\right) \tilde{N}(d t, d e) .
\end{gathered}
$$

Where, to simplify we have taken $d=q=q^{\prime}=1$ and $W=B$ and we have used the fact that $V\left(t^{-}, z\right)=X_{t^{-}}\left(Y_{t^{-}}(z)\right)$ and the notation

$$
\bar{V}(t, y, e):=X_{t^{-}}\left(Y_{t^{-}}(y)+g\left(t, Y_{t^{-}}(y), e\right)\right)+h\left(t, Y_{t^{-}}(y)+g\left(t, Y_{t^{-}}(y), e\right)\right) .
$$

$\bar{V}(t, ., e)$ corresponds to $V(t,$.$) after a jump on (t, e)$ which is the compound of $X_{t^{-}}()+$. $h\left(t, X_{t^{-}}(), e.\right)$ corresponding to $X$ after a jump on $(t, e)$ and $Y_{t^{-}}()+.g\left(t, Y_{t^{-}}(), e.\right)$ corresponding to $Y$ after a jump at the same time for the same mark $e$.

In the reverse direction, i.e., linking SPDE with SDEs is not obvious but it is possible in the cases considered by H. Kunita [Kun97, Chapter 6] and utility-SPDEs of [EM13, MM20], see Section 4 below.

\subsection{Preliminary results}

In the following we will often need to control in $L_{p}$-norms of a $d$-dimensional semimartingales $Z_{t}=\left(Z_{t}^{1}, Z_{t}^{2}, \ldots, Z_{t}^{d}\right)$ solutions of a stochastic differential equation of the following form

$$
Z_{t}^{i}=z^{i}+\int_{0}^{t} b_{s}^{i} d s+\sum_{j=1}^{m} \int_{0}^{t} f^{i, j} d \hat{W}_{s}^{j}+\int_{0}^{t} \int_{\mathbb{R}^{q^{\prime}}} g^{i}(s, e) \tilde{N}(d t, d e) .
$$

where $\hat{W}$ be a $m$-dimensional brownian motion and $Z_{0}=z \in \mathbb{R}^{d}$. To do, we use the following result established in [Kun04, Theorem 2.11]

Theorem 3. For any $p \geq 2$, there exists a positive constant $C_{p}$ such that

$$
\begin{aligned}
\mathbb{E}\left[\sup _{0<s \leq t}\left|Z_{s}\right|^{p}\right] & \leq C_{p}\left\{|z|^{p}+\mathbb{E}\left[\left(\int_{0}^{t}\left|b_{s}\right| d s\right)^{p}\right]+\mathbb{E}\left[\left(\int_{0}^{t}\left|f_{s}\right|^{2} d s\right)^{\frac{p}{2}}\right]\right. \\
+ & \left.\mathbb{E}\left[\left(\int_{0}^{t} \int_{\mathbb{R}^{q^{\prime}}}|g(s, e)|^{2} \nu(d e) d s\right)^{\frac{p}{2}}\right]+\mathbb{E}\left[\int_{0}^{t} \int_{\mathbb{R}^{q^{\prime}}}|g(s, e)|^{p} \nu(d e) d s\right]\right\} .
\end{aligned}
$$

In this work, the aim is not to improve the simulation methods or to propose a new scheme for SDEs with jumps or to work in the most general framework possible, see for example [PrTa97, [BrPl07], [MS08] and [KoTa10] and their references. Here we study the convergence of the compound of two numerical schemes. Our results show that it is enough to approximate the solutions of two SDEs to obtain an approximation of the solution of a SPDE. What is interesting in this work is the convergence result and particularly the rate of convergence which we obtain for the SPDE from those of the two SDEs. At the same time this avoids us to directly discretize this class of equations, which requires inevitably to approximate $U_{z}(t, z)$ and $U_{z z}(t, z)$, using a finite differences method for example, which requires the resolution in the full space (or on a grid in $z$ ). This is computationally demanding. In addition, it seems really difficult to obtain error estimates in that context. To simplify the presentation, we will assume in the following that $\nu\left(\mathbb{R}^{q^{\prime}}\right)<\infty$ which implies, by the Jenssen inequality

$$
\mathbb{E}\left[\left(\int_{0}^{t} \int_{\mathbb{R}^{q^{\prime}}}|g(s, e)|^{2} \nu(d e) d s\right)^{\frac{p}{2}}\right] \leq t^{\frac{p}{2}-1}\left[\nu\left(\mathbb{R}^{q^{\prime}}\right)\right]^{\frac{p}{2}-1} \mathbb{E}\left[\int_{0}^{t} \int_{\mathbb{R}^{q^{\prime}}}|g(s, e)|^{p} \nu(d e) d s\right] .
$$

So a particular case of this last result is the following Corollary, useful in the sequel. 
Corollary 2. Assume $\nu\left(\mathbb{R}^{q^{\prime}}\right)<\infty$, then for any $p \geq 2$, there exists a positive constant $C_{p}$ such that the solution $Z$ of (11) satisfies

$$
\begin{array}{r}
\mathbb{E}\left[\sup _{0<s \leq t}\left|Z_{s}\right|^{p}\right] \leq C_{p}\left\{|z|^{p}+\mathbb{E}\left[\left(\int_{0}^{t}\left|b_{s}\right| d s\right)^{p}\right]+\mathbb{E}\left[\left(\int_{0}^{t}\left|f_{s}\right|^{2} d s\right)^{\frac{p}{2}}\right]\right. \\
\left.+\mathbb{E}\left[\int_{0}^{t} \int_{\mathbb{R}^{q^{\prime}}}|g(s, e)|^{p} \nu(d e) d s\right]\right\} .
\end{array}
$$

To obtain more precise estimates, which are necessary for our study, we need to specify the regularity assumptions on the SDE's coefficients.

\subsection{Assumptions}

For the rest of the paper, the process $X$ plays the role of the random field $F$ in Theorem 1. It must therefore verify most of the assumptions of this result, namely $(\mathbf{H 1} \mathbf{- H 2}-\mathbf{H} 3)$ and for this, we state related assumptions on the $\mathbb{R}^{d}$-valued drift coefficient $\mu=\left\{\mu(t, x) ; t \in[0, T], x \in \mathbb{R}^{d}\right\}$,

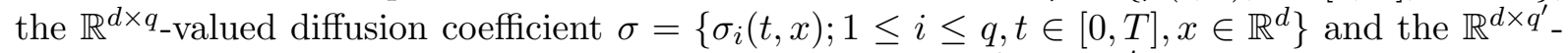
jump coefficient $h=\left\{h_{i}(t, x, e) ; 1 \leq i \leq q, t \in[0, T], x \in \mathbb{R}^{d}, e \in \mathbb{R}^{q^{\prime}}\right\}$ which we suppose to be regular enough in time and space. When we will discuss on approximation of $X(Y)$, similar assumptions will be made on the coefficients $b$ and $\gamma_{i}$ of Equation (9) for $Y$.

(HP1) The coefficients $\mu, \sigma$ and $h$ are Lipschitz continuous with respect to the space variable $x$, uniformly in time. More precisely, there exist positive constants $C^{X}$ and $C^{X}(e)$ such that for any $t \in[0, T], x, y \in \mathbb{R}^{d}$ and $e \in \mathbb{R}$

$$
\left\{\begin{array}{l}
|\mu(t, x)-\mu(t, y)| \leq C^{X}|x-y|, \quad|\mu(t, 0)| \leq C^{X} \\
|\sigma(t, x)-\sigma(t, y)| \leq C^{X}|x-y|, \quad|\sigma(t, 0)| \leq C^{X} \\
|h(t, x, e)-h(t, y, e)| \leq C^{X}(e)|x-y|, \quad|h(t, 0, e)| \leq C^{X}(e),
\end{array}\right.
$$

where, the constant $C^{X}(e)$ satisfies $\int_{\mathbb{R}^{q^{\prime}}}\left[C^{X}(e)\right]^{p} \nu(d e)<\infty, \forall p \geq 2$.

(HP2) $\mu, \sigma$ and $h$ are continuously differentiable with respect to the space variable $x$ such that their derivatives $\nabla_{x} \mu:=\left\{\nabla_{x} \mu(t, x) ; t \in[0, T], x \in \mathbb{R}^{d}\right\}, \nabla_{x} \sigma=\left\{\nabla_{x} \sigma_{i}(t, x) ; 1 \leq i \leq q, t \in\right.$ $\left.[0, T], x \in \mathbb{R}^{d}\right\}$ and $\nabla_{x} h:=\left\{\nabla_{x} h(t, x, e) ; t \in[0, T], x \in \mathbb{R}^{d}, e \in \mathbb{R}^{q^{\prime}}\right\}$ are $\delta$-Hölder for a certain exponent $\delta \in(0,1]$. Namely, there exist positive constants $C^{X, \nabla}$ and $C^{X, \nabla}(e)$ such that for any $t \in[0, T], x, y \in \mathbb{R}^{d}$ and $e \in \mathbb{R}^{q^{\prime}}$

$$
\left\{\begin{array}{l}
\left|\nabla_{x} \mu(t, x)-\nabla_{x} \mu(t, y)\right| \leq C^{X, \nabla}|x-y|^{\delta}, \quad\left|\nabla_{x} \mu(t, x)\right| \leq C^{X, \nabla} \\
\left|\nabla_{x} \sigma(t, x)-\nabla_{x} \sigma(t, y)\right| \leq C^{X, \nabla}|x-y|^{\delta}, \quad\left|\nabla_{x} \sigma(t, x)\right| \leq C^{X, \nabla}, \\
\left|\nabla_{x} h(t, x, e)-\nabla_{x} h(t, y, e)\right| \leq C^{X, \nabla}(e)|x-y|^{\delta},\left|\nabla_{x} h(t, x, e)\right| \leq C^{X, \nabla}(e)
\end{array}\right.
$$

with $\int_{\mathbb{R}^{q^{\prime}}}\left[C^{X, \nabla}(e)\right]^{p} \nu(d e)<\infty, \forall p \geq 2$.

(HP3) $\mu, \sigma$ and $h$ are Hölder continuous in time, locally in space, i.e. there exists an exponent $\alpha \in(0,1]$ and a positive constants $C^{X}$ and $C^{X}(e)\left(\int_{\mathbb{R}^{q^{\prime}}} C^{X}(e)^{p} \nu(d e)<\infty, \forall p \geq 2\right)$, such that for any $x \in \mathbb{R}^{d}, e \in \mathbb{R}$ and $s, t \in[0, T]$

$$
\left\{\begin{array}{l}
|\mu(t, x)-\mu(s, x)|+|\sigma(t, x)-\sigma(s, x)| \leq C^{X}(1+|x|)|t-s|^{\alpha} \\
|h(t, x, e)-h(s, x, e)| \leq C^{X}(e)(1+|x|)|t-s|^{\alpha}
\end{array}\right.
$$


(HP4) $\nabla_{x} \mu, \nabla_{x} \sigma$ and $\nabla_{x} h$ are Hölder continuous in time, locally in space, i.e. there exists an exponent $\alpha \in(0,1]$, such that for any $x \in \mathbb{R}^{d}, e \in \mathbb{R}$ and $s, t \in[0, T]$

$$
\left\{\begin{array}{l}
\left|\nabla_{x} \mu(t, x)-\nabla_{x} \mu(s, x)\right|+\left|\nabla_{x} \sigma(t, x)-\nabla_{x} \sigma(s, x)\right| \leq C^{X, \nabla}(1+|x|)|t-s|^{\alpha} . \\
\left|\nabla_{x} h(t, x, e)-\nabla_{x} h(s, x, e)\right| \leq C^{X, \nabla}(e)(1+|x|)|t-s|^{\alpha} .
\end{array}\right.
$$

Assumption (HP1) ensures the existence of a strong solution to the $\operatorname{SDE}(\mu, \sigma, h, W, N)$ Kun04, Theorem 3.1] and plays a crucial role to establish a $\mathbf{L}_{p}$-estimates. It is also well-known [Kun04, Theorem 3.2] combined with Kolmogorov-Totoki's Theorem [Kun04, Theorem 4.1], that the map $(t, x) \mapsto X_{t}(\omega, x)$ has a modification $X^{\prime}$ such that for any $x$ it is càdlàg with respect to $t$ and for any $t$ it is continuous in $x$ a.s., we shall systematically work with this modification from now on. Assumption $(\overline{\text { HP2 }}$ ) is a sufficient condition (see Kun04, Theorem 3.4]) under which the above map is $C^{1}$ in $x$. Assumptions (HP3) and (HP4) enable us, essentially, to establish convergence results of the Euler discretization scheme within the paper setting.

\subsection{SDE: differentiability, local and uniform estimates}

To analyze the approximation of the compound maps $X \circ Y$, precise estimates on the maps $x \mapsto X_{t}(\omega, x)$ are needed: Such random fields are also called stochastic flows and are the main subject of Kunita's book and papers [Kun97, Kun04, FuKun85, see also [Kun97, Chapter 3 and 4] for framework without jumps. As aforementioned, under (HP1), the map $(t, x) \mapsto X_{t}(\omega, x)$ has a good modification, continuous with respect to the spatial paramter Kun04, Theorem $3.2]$, we are working with. The additional space regularity is connected to the regularity of the coefficients $(\mu, \sigma, h)$ as it is shown in [Kun04, Theorem 3.4]. Indeed, under Assumptions (HP1) and (HP2), the strong solution $X_{t}(x)$ to 8 is continuously differentiable in space and its derivative denoted by $\nabla X_{t}(x)$ is locally $\varepsilon$-Hölder ${ }^{1}$ for any $\varepsilon<\delta$. Furthermore, it is a semimartingale solution of a linear equation, with bounded stochastic parameters $\left(\nabla_{x} \mu\left(t, X_{t}(x)\right), \nabla_{x} \sigma\left(t, X_{t}(x)\right), \nabla_{x} h\left(t, X_{t}(x), e\right)\right)$ given by

$$
\left\{\begin{aligned}
\mathrm{d} \nabla X_{t}(x) & =\nabla_{x} \mu\left(t, X_{t}(x)\right) \nabla X_{t}(x) \mathrm{d} t+\sum_{i=1}^{q} \nabla_{x} \sigma_{i}\left(t, X_{t}(x)\right) \nabla X_{t}(x) \mathrm{d} W_{t}^{i} \\
& +\int_{\mathbb{R}} \nabla_{x} h\left(t, X_{t^{-}}(x), e\right) \nabla X_{t^{-}}(x) \tilde{N}(d t, d e), \\
\nabla X_{0}(x) & =\mathrm{Id} .
\end{aligned}\right.
$$

We now proceed to $L_{p}$-estimates of $X_{t}(x)$ and its derivative $\nabla_{x} X_{t}(x)$. We collect several useful results, some of them are showed in the literature, in the following Proposition.

Proposition 1. Assume (HP1). For any $p>0$, there exist generic constants $C_{p, 13}$ and $C_{p, 14}$ such that

$$
\begin{aligned}
&\left\|X_{t}(x)\right\|_{\mathbf{L}_{p}} \leq C_{p, \sqrt[13]{13}}(1+|x|), \\
&\left\|X_{t}(x)-X_{t}(y)\right\|_{\mathbf{L}_{p}} \leq C_{p,[14}|x-y|,
\end{aligned}
$$

for any $(t, x, y) \in[0, T] \times \mathbb{R}^{d} \times \mathbb{R}^{d}$. In addition under $(\mathbf{H P 2})$, for any $p>0$ there exist generic constants $C_{p, 15}$ and $C_{p, 16}$ such that

$$
\left\|\nabla X_{t}(x)\right\|_{\mathbf{L}_{p}} \leq C_{p, \sqrt{15},}
$$

\footnotetext{
${ }^{1}$ That is for any compact $K$ of $\mathbb{R}^{d}$ there exists a finite positive random variable $C(K)$ such that for any $x, y \in K$ we have $\left|\nabla X_{t}(x, \omega)-\nabla X_{t}(y, \omega)\right| \leq C(K, \omega)|x-y|^{\varepsilon}$ a.s., see [Kun04, Theorem 3.3] for details .
} 


$$
\left\|\nabla X_{t}(x)-\nabla X_{t}(y)\right\|_{\mathbf{L}_{p}} \leq C_{p,[16}|x-y|^{\delta}
$$

for any $(t, x, y) \in[0, T] \times \mathbb{R}^{d} \times \mathbb{R}^{d}$.

Proof. The proofs of inequalities (13) and (14) are given by Theorem 3.2 of Kunita's paper Kun04. The uniform estimate (15) is also easy to obtain, in view of 112 and owing to the boundedness of $\nabla_{x} \mu, \nabla_{x} \sigma_{i}$ and $\nabla_{x} h$, we leave the details to the reader.

Let show (16) under ( $\mathbf{\text { HP2 }}$. For simplicity, we provide the proof when $d=q=1$, the general case being similar. Also, we focus on the case $p \geq 2$ since we can deduce the result for $p<2$ using the stability of $\mathbf{L}_{p}$-norm ${ }^{2}$ combined with the result for $p=2$. First, from (12) write

$$
\begin{aligned}
\nabla X_{t}(x)-\nabla X_{t}(y) & =\int_{0}^{t}\left(\nabla_{x} \mu\left(s, X_{s}(x)\right) \nabla X_{s}(x)-\nabla_{x} \mu\left(s, X_{s}(y)\right) \nabla X_{s}(y)\right) \mathrm{d} s \\
& +\int_{0}^{t}\left(\nabla_{x} \sigma\left(s, X_{s}(x)\right) \nabla X_{s}(x)-\nabla_{x} \sigma\left(s, X_{s}(y)\right) \nabla X_{s}(y)\right) \mathrm{d} W_{s} \\
& +\int_{0}^{t} \int_{\mathbb{R}}\left(\nabla_{x} h\left(s, X_{s^{-}}(x), e\right) \nabla X_{s^{-}}(x)-\nabla_{x} h\left(s, X_{s^{-}}(y), e\right) \nabla X_{s^{-}}(y)\right) \tilde{N}(d s, d e) .
\end{aligned}
$$

Take the power $p$ and the expectation, and apply Corollary 2 , we get for some constante $C_{p}$,

$$
\begin{gathered}
\mathbb{E}\left[\left|\nabla X_{t}(x)-\nabla X_{t}(y)\right|^{p}\right] \leq C_{p}\left\{\mathbb{E}\left[\left(\int_{0}^{t}\left|\nabla_{x} \mu\left(s, X_{s}(x)\right) \nabla X_{s}(x)-\nabla_{x} \mu\left(s, X_{s}(y)\right) \nabla X_{s}(y)\right| d s\right)^{p}\right]\right. \\
+\mathbb{E}\left[\left(\int_{0}^{t}\left|\nabla_{x} \sigma\left(s, X_{s}(x)\right) \nabla X_{s}(x)-\nabla_{x} \sigma\left(s, X_{s}(y)\right) \nabla X_{s}(y)\right|^{2} d s\right)^{\frac{p}{2}}\right] \\
\left.+\mathbb{E}\left[\int_{0}^{t} \int_{\mathbb{R}}\left|\nabla_{x} h\left(s, X_{s^{-}}(x), e\right) \nabla X_{s^{-}}(x)-\nabla_{x} h\left(s, X_{s^{-}}(y), e\right) \nabla X_{s^{-}}(y)\right|^{p} \nu(d e) d s\right]\right\} .
\end{gathered}
$$

Now write,

$$
\begin{aligned}
\nabla_{x} \mu\left(s, X_{s}(x)\right) \nabla X_{s}(x)-\nabla_{x} \mu\left(s, X_{s}(y)\right) \nabla X_{s}(y) & =\nabla_{x} \mu\left(s, X_{s}(x)\right)\left(\nabla X_{s}(x)-\nabla X_{s}(y)\right) \\
& +\left(\nabla_{x} \mu\left(s, X_{s}(s)\right)-\nabla_{x} \mu\left(s, X_{s}(y)\right)\right) \nabla X_{s}(y) .
\end{aligned}
$$

Then applying twice the Jenssen inequality, one gets

$$
\begin{aligned}
\mathbb{E} & {\left[\left(\int_{0}^{t}\left|\nabla_{x} \mu\left(s, X_{s}(x)\right) \nabla X_{s}(x)-\nabla_{x} \mu\left(s, X_{s}(y)\right) \nabla X_{s}(y)\right| d s\right)^{p}\right] } \\
\leq & 2^{p-1} t^{p-1}\left\{\int_{0}^{t} \mathbb{E}\left[\left|\nabla_{x} \mu\left(s, X_{s}(x)\right)\left(\nabla X_{s}(x)-\nabla X_{s}(y)\right)\right|^{p}\right] d s\right. \\
& \left.+\int_{0}^{t} \mathbb{E}\left[\left|\left(\nabla_{x} \mu\left(s, X_{s}(s)\right)-\nabla_{x} \mu\left(s, X_{s}(y)\right)\right) \nabla X_{s}(y)\right|^{p}\right] d s\right\} .
\end{aligned}
$$

Now, take advantage of the Assumptions ( and (15): it readily follows that

$$
\begin{gathered}
\mathbb{E}\left[\left(\int_{0}^{t}\left|\nabla_{x} \mu\left(s, X_{s}(x)\right) \nabla X_{s}(x)-\nabla_{x} \mu\left(s, X_{s}(y)\right) \nabla X_{s}(y)\right| d s\right)^{p}\right] \\
\leq 2^{p-1} T^{p-1}\left[C^{X, \nabla}\right]^{p}\left\{\int_{0}^{t} \mathbb{E}\left[\left|\nabla X_{s}(x)-\nabla X_{s}(y)\right|^{p}\right] d s+T C_{p,[15} C_{p \delta,[14]}^{p \delta}|x-y|^{p \delta}\right\} .
\end{gathered}
$$

${ }^{2}$ It writes $\|Z\|_{\mathbf{L}_{p}} \leq\|Z\|_{\mathbf{L}_{q}}$ for any $0<p \leq q$. 
The same reasoning and Assumptions lead us to deduce that

$$
\begin{gathered}
\mathbb{E}\left[\left(\int_{0}^{t}\left|\nabla_{x} \sigma\left(s, X_{s}(x)\right) \nabla X_{s}(x)-\nabla_{x} \sigma\left(s, X_{s}(y)\right) \nabla X_{s}(y)\right|^{2} d s\right)^{\frac{p}{2}}\right] \\
\leq 2^{p-1} T^{\frac{p}{2}-1}\left[C^{X, \nabla}\right]^{p}\left\{\int_{0}^{t} \mathbb{E}\left[\left|\nabla X_{s}(x)-\nabla X_{s}(y)\right|^{p}\right] d s+T C_{p, \sqrt{15})} C_{p \delta,[14}^{p \delta}|x-y|^{p \delta}\right\} .
\end{gathered}
$$

and

$$
\begin{gathered}
\mathbb{E}\left[\int_{0}^{t} \int_{\mathbb{R}}\left|\nabla_{x} h\left(t, X_{t^{-}}(x), e\right) \nabla X_{t^{-}}(x)-\nabla_{x} h\left(t, X_{t^{-}}(y), e\right) \nabla X_{t^{-}}(y)\right|^{p} \nu(d e) d s\right] \\
\leq 2^{p-1} T^{p-1}\left(\int_{\mathbb{R}}\left[C^{X, \nabla}(e)\right]^{p} \nu(d e)\right)\left\{\int_{0}^{t} \mathbb{E}\left[\left|\nabla X_{s}(x)-\nabla X_{s}(y)\right|^{p}\right] d s+T C_{p,[15]} C_{p \delta,[14}^{p \delta}|x-y|^{p \delta}\right\}_{20)}
\end{gathered}
$$

Injecting (18), (19) and (20) in (17), the quantity $\iota(t):=\mathbb{E}\left(\left|\nabla X_{t}(x)-\nabla X_{t}(y)\right|^{p}\right)$ satisfies

$$
\left\{\begin{array}{l}
\iota(t) \leq C_{p, 1}(T)\left(\int_{0}^{t} \iota(s) \mathrm{d} s+C_{p, 2}(T)|x-y|^{p \delta}\right), \\
C_{p, 1}(T):=2^{p-1} C_{p} T^{p / 2-1}\left(\left[C^{X, \nabla}\right]^{p}\left(1+T^{p / 2}\right)+\int_{\mathbb{R}}\left[C^{X, \nabla}(e)\right]^{p} \nu(d e)\right), \\
C_{p, 2}(T):=T C_{p,[15]} C_{p \delta,[14]}^{p \delta} .
\end{array}\right.
$$

The estimate 16 is then a direct consequence of Gronwall's lemma.

We now combine Theorem 2 , Corollary 1 with Proposition 1 to put the sup over the space variable inside the expectation in Proposition 1. This is the following assertion, which is a new result to our knowledge.

Theorem 4. Let Assumption (HP1) holds. For any $p>0$ and any $\beta \in(0,1)$, there exist generic constants $C_{p, 21]}$ and $C_{p, 22]}$ such that, for any $t \in[0, T]$,

$$
\begin{gathered}
\left\|\sup _{|x| \leq \lambda}\left|X_{t}(x)\right|\right\|_{\mathbf{L}_{p}} \leq C_{p, 221} \lambda, \quad \forall \lambda \geq 1, \\
\left\|\sup _{x \neq y,|x| \leq \lambda,|y| \leq \lambda} \frac{\left|X_{t}(x)-X_{t}(y)\right|}{|y-x|^{\beta}}\right\|_{\mathbf{L}_{p}} \leq C_{p,[22} \lambda^{1-\beta}, \quad \forall \lambda \geq 1 .
\end{gathered}
$$

If in addition (HP2) is satisfied. For any $p>0$ and any $\beta \in(0, \delta)$, there exist generic constants $C_{p, 23]}, C_{p, 24]}$ and $C_{p, 25}$ such that, for any $t \in[0, T]$,

$$
\begin{array}{r}
\left\|\sup _{|x| \leq \lambda}\left|\nabla X_{t}(x)\right|\right\|_{\mathbf{L}_{p}} \leq C_{p, 23\}} \lambda^{\delta}, \quad \forall \lambda \geq 1, \\
\left\|\sup _{x \neq y,|x| \leq \lambda,|y| \leq \lambda} \frac{\left|\nabla X_{t}(x)-\nabla X_{t}(y)\right|}{|y-x|^{\beta}}\right\|_{\mathbf{L}_{p}} \leq C_{p, 224} \lambda^{\delta-\beta}, \quad \forall \lambda \geq 1, \\
\left\|\sup _{x \neq y,|x| \leq \lambda,|y| \leq \lambda} \frac{\left|X_{t}(x)-X_{t}(y)\right|}{|y-x|}\right\|_{\mathbf{L}_{p}} \leq C_{p, 25)} \lambda^{\delta}, \quad \forall \lambda \geq 1 .
\end{array}
$$

Observe that the additional smoothness in $(\overline{\text { HP2 }})$ enables us to improve $(22)$ (for $\beta<1$ ) to (25) (i.e. $\beta=1$ ): this improvement will play an important role in the derivation of our main Theorem 5, with the optimal convergence order $\beta$. 
Proof. Let $\beta \in(0,1)$ : We first show (21) and $(22)$ for any $p>d /(1-\beta)>d$. Owing to (14), we can apply Theorem 2 to $G(x):=X_{t}(x)$ with $\beta^{(G)}=1 \in(d / p, 1]$ and $\tau^{(G)}=0$, to conclude that (22) holds with the given index $\beta$ since $\beta<1-d / p \Leftrightarrow p>d /(1-\beta)$. Moreover the application of Corollary 1 provides (21). Now we relax the constraint on $p$ : for $p \leq d /(1-\beta)$, set $\bar{p}=2 d /(1-\beta)$ for which $(22)$ holds and use the stability property of $\mathbf{L}_{p}$-norm to write

$$
\begin{aligned}
\left\|\sup _{x \neq y,|x| \leq \lambda,|y| \leq \lambda} \frac{\left|X_{t}(x)-X_{t}(y)\right|}{|y-x|^{\beta}}\right\|_{\mathbf{L}_{p}} & \leq\left\|\sup _{x \neq y,|x| \leq \lambda,|y| \leq \lambda} \frac{\left|X_{t}(x)-X_{t}(y)\right|}{|y-x|^{\beta}}\right\|_{\mathbf{L}_{\bar{p}}} \\
& \leq C_{\bar{p},\left[22 \lambda^{1-\beta} .\right.}
\end{aligned}
$$

The same arguments apply to prove that (21) holds for any $p>0$.

The justification of (23) and (24) follows the same arguments as above, using (16) instead of (14): then Corollary 1 and Theorem 2 can be applied to $G(x):=\nabla X_{t}(x)$ with $\beta^{(G)}=\delta$ and $\tau^{(G)}=0$. We leave the details to the reader.

Last, observe that for any $x, y$ such that $|x| \leq \lambda$ and $|y| \leq \lambda$, we have $\left|X_{t}(x)-X_{t}(y)\right| \leq$ $\sup _{|z| \leq \lambda}\left|\nabla X_{t}(z)\right||y-x|$ : thus, 25) readily follows from 23].

Remark 3.1. As a direct consequence of this Theorem: If the coefficients of X satisfy (HP1) and (HP3), then

1. Assumption $(\mathbf{H 1})$ is satisfied with $C_{p}^{\sqrt{\mathbf{H 1} 1}}:=C_{p, 21}$ and $\alpha_{p}^{\sqrt{\mathbf{H 1} 1}}:=1$ in view of Theorem 4 .

2. Assumption $(\mathbf{H 2})$ is satisfied for any given $\kappa \in(0,1)$ with $C_{p}^{\sqrt{\mathbf{H 2}}}:=C_{p, \sqrt{22})}$ (depending on $\kappa)$ and $\alpha \stackrel{\mathrm{H2}}{\mathbf{H}}:=1-\kappa$.

Before stating our main result, it remains to show Assumptions $(\mathbf{H 2}-\mathbf{H 3})$, but for that we need first to introduce the Euler schemes and study their local and uniform estimates.

\subsection{Compound Euler schemes: Main result}

In order to give the Euler scheme, we first expose how to simulate the integral with respect to the Lévy measure. We follow the presentation of [MS08.

Simulation of the integral with respect to the poisson random measure Consider a sequence $e_{1}, e_{2}, \ldots$ of independent random variables with common exponential distribution with parameter 1. Define

$$
\Lambda(t)=\int_{0}^{t} \lambda(s) d s, t \in[0, T]
$$

The number of jumps of the random Poisson measure $\tilde{N}(d t, d e)$ in an interval $[0, t]$ is determined as

$$
J(t)=\max \left\{\sum_{j=1}^{k} e_{j} \leq \Lambda(t)\right\},
$$

and the total number of jumps in $[0, T]$ is denoted by $J=J(T)$. The jump times of the Poisson measure can be defined by $\theta_{0}=0$,

$$
\theta_{k}=\Lambda^{-1}\left(\max \left\{\sum_{j=1}^{k} e_{j}\right\}\right), k \in \llbracket 1, J \rrbracket,
$$


and can be computed recursively by

$$
e_{k}=\int_{\theta_{k-1}}^{\theta_{k}} \lambda(s) d s, k \in \llbracket 1, J \rrbracket .
$$

Once the jump times are computed, we proceed to sample the marks $\left\{E_{k}\right\}$, that, conditionally on the values of the jumps times, are independent random variables distributed respectively according to $\{\nu(d e)\}$. The random measure with intensity $\lambda(t) d t \times \nu(d e)$ can then be constructed as

$$
N(d t, d e)=\sum_{k=1}^{J} \delta_{\left(\theta_{k}, E_{k}\right)}(d t, d e)
$$

and, consequently, the stochastic integral with respect to the Poisson random measure, i.e. the last term in the SDE (8), can be computed as

$$
\int_{0}^{t} \int_{\mathbb{R}} h\left(s, X_{s^{-}}, e\right) N(d s, d e)=\sum_{k=1}^{J(t)} h\left(\theta_{k}, X_{\theta_{k}^{-}}, E_{k}\right), t \in[0, T] .
$$

Euler Scheme Let $N \geq 1$ and consider the discretization family $\left\{\bar{t}_{i}:=i \frac{T}{N}, i \in \llbracket 0, N \rrbracket\right\}$ of $[0, T]$. Consider also the jump times $\left\{\theta_{k}, k \in \llbracket 1, J \rrbracket\right\}$ with corresponding marks $\left\{E_{k}, k \in \llbracket 1, J \rrbracket\right\}$ as explained in MS08]. Consider the augmented partition given by the union

$$
\left\{t_{l}, l \in \llbracket 0, N+J \rrbracket\right\}:=\left\{\bar{t}_{i}:=i \frac{T}{N}, i \in \llbracket 0, \bar{N} \rrbracket\right\} \cup\left\{\theta_{k}, k \in \llbracket 1, J \rrbracket\right\}
$$

- Set $X_{0}^{N}(x)=x$.

- For $k=0, \ldots, N+J-1$ and $t \in\left(t_{k}, t_{k+1}\right]$, set

$$
\begin{aligned}
X_{t^{-}}^{N}(x) & =X_{t_{k}}^{N}(x)+\mu\left(t_{k}, X_{t_{k}}^{N}(x)\right)\left(t-t_{k}\right)+\sum_{i=1}^{q} \sigma_{i}\left(t_{k}, X_{t_{k}}^{N}(x)\right)\left(W_{t}^{i}-W_{t_{k}}^{i}\right) \\
& -\left(t-t_{k}\right) \lambda\left(t_{k}\right) \int_{\mathbb{R}} h\left(t_{k}, X_{t_{k}}^{N}(x), e\right) \nu(d e) .
\end{aligned}
$$

- When $t_{k+1}=\theta_{l}$, we introduce a correction due to jump discontinuities.

$$
X_{t_{k+1}}^{N}(x)=X_{t_{k+1}^{-}}^{N}(x)+h\left(\theta_{l}, X_{\theta_{l}^{-}}^{N}(x), E_{l}\right) .
$$

It can be equivalently written for any time $t \in[0, T]$ : Denoting by $\tau_{t}:=\max \{k \in \llbracket 0, N+$ $\left.J \rrbracket: t_{k} \leq t\right\}$ the last discretization-time before $t$, we have

$$
\begin{aligned}
X_{t}^{N}(x) & =x+\int_{0}^{t} \mu\left(\tau_{s}, X_{\tau_{s}}^{N}(x)\right) \mathrm{d} s+\sum_{i=1}^{q} \int_{0}^{t} \sigma_{i}\left(\tau_{s}, X_{\tau_{s}}^{N}(x)\right) \mathrm{d} W_{s}^{i} \\
& +\int_{0}^{t} \int_{\mathbb{R}} h\left(\tau_{s}, X_{\tau_{s}^{-}}^{N}, e\right) \tilde{N}(d s, d e) .
\end{aligned}
$$

Where the last integral is as in (26). Similarly, assume that $b, \gamma$ and $g$ fulfill (HP1), so that the strong solution $Y$ to $(9)$ is well defined, together with its Euler scheme $Y^{N}$. 
Remark 3.2. Here we consider the Euler scheme for the simple reason that it is the simplest and the best known scheme to discretize an SDE, see fro example [PrTa97] and [BrPl07]. As we can read in Theorem 1, the result is true for any approximation considered for $F$ and $\Theta$, but we have to expect that the convergence rate of the compound of the approximations changes; the convergence rate for Euler scheme is different from the one of Milstein. It will be interesting to study what happens if we consider the last one or other scheme, this will be considered in a forthcoming work.

The rest of the section is devoted to establish the following main result.

Theorem 5 (Main). Assume that $(\mu, \sigma, h)$ satisfies Assumptions (IP1) and (HP3) (which $\alpha$ parameter is denoted by $\left.\alpha^{X}\right)$ and that $(b, \gamma, g)$ satisfies Assumptions (HP1) and (HP3) (which $\alpha$-parameter is denoted by $\alpha^{Y}$ ).

Then the compound Euler scheme $X_{.}^{N}\left(Y^{N}\right)$ converges to $X .\left(Y\right.$.) in any $\mathbf{L}_{p}$-norm, at the order almost equal to $\beta:=\min \left(\alpha^{X}, \alpha^{Y}, \frac{1}{2}\right)$ w.r.t. $N$ : For any $p>0$ and any $\rho>0$, there is a finite constant $C_{p, \rho}$ such that for any $s, t \in[0, T]$,

$$
\left\|X_{t}^{N}\left(Y_{s}^{N}\right)-X_{t}\left(Y_{s}\right)\right\|_{\mathbf{L}_{p}} \leq C_{p, \rho} N^{-\beta+\rho}, \quad \forall N \geq 1 .
$$

Assume additionally that $(\mu, \sigma, h)$ satisfies Assumptions $(\mathbf{H P 2})$ and $(\mathbf{H P 4})$ with the $\alpha$-parameter equal to $\alpha^{X}$. Then the above estimate holds true with $\rho=0$, i.e. $X^{N}\left(Y_{.}^{N}\right)$ converges to $X .(Y$. in any $\mathbf{L}_{p}$-norm at the optimal order $\beta$.

\subsection{Proof of Theorem 5}

The proof of this result requires several intermediate results, some of them being completely new (Theorems 4 and 7 and Proposition 3). Since Theorem 4 above implies $(\overline{\mathbf{H 1}})-(\overline{\mathbf{H} 2})$ (see Remark 3.1), we seek to prove that Assumptions $(\mathbf{H 3})-(\mathbf{H} 4-\mathrm{a})$ are also satisfied if that of Theorem 5 hold true.

Throughout this proof, we will make use of different constants that may depend on the integer $p$ of $\mathbf{L}_{p}$-norm, on the dimensions $d$ and $q$, on the time horizon $T$ and on the constants from the assumptions: These constants will be called generic constant and will be denoted by the same notation $C_{p}$ even if their values change from line to line. They will not depend on $N$.

We denote by $C_{p}^{\text {BDG }}$ the constant of the upper Burkholder's inequality with $\mathbf{L}_{p}$-norm, see Ikeda-Watanabe [IkWa81].

\subsubsection{Euler scheme: local and uniform estimates}

In order to prove Theorem 5 , we partly generalize the previous results about the SDE to its Euler approximation. Some derivations are more subtle and require details at some places. Recall the definition of Euler scheme in (28).

First, as for the solution of the $\operatorname{SDE}(\mu, \sigma)$, some estimates for its approximation scheme are needed. This is the analogue of Proposition 1 given in the first statement of the next Proposition. Second, using the same arguments than for the SDE case (Theorem 4), we can put the sup over the space variable inside the $\mathbf{L}_{p}$-norm to derive the second statement of the following result.

Proposition 2. Let (HP1) hold true.

\footnotetext{
${ }^{3}$ The paper [BrPl07] is a survey of strong discrete time approximations of jump-diffusion processes described by SDEs.
} 
(i) For any $p>0$ there exist generic constants $C_{p, 29]}$ and $C_{p, 30}$ such that

$$
\begin{aligned}
& \left\|X_{t}^{N}(x)\right\|_{\mathbf{L}_{p}} \leq C_{p, 29\}}(1+|x|), \\
& \left\|X_{t}^{N}(x)-X_{t}^{N}(y)\right\|_{\mathbf{L}_{p}} \leq C_{p, 30\}}|x-y|,
\end{aligned}
$$

for any $(t, x, y) \in[0, T] \times \mathbb{R}^{d} \times \mathbb{R}^{d}$.

(ii) The estimates (21) and 22) where we replace $X$ by $X^{N}$ hold true, up to changing the generic constants.

We omit the proof which is quite standard, see that of Theorem 3.2 of Kunita's paper Kun04. Let us now show the following estimates on local increments, it will be needed for the sequel.

Lemma 1. Let Assumption (HP1) holds and let $p>0$. Then there exist generic constants $C_{p, \text {,31] }}$ and $C_{p, 32]}$ such that, for any $x, y \in \mathbb{R}^{d}$ and any $t \in[0, T]$,

$$
\begin{array}{r}
\left\|\sup _{\tau_{t} \leq u \leq t}\left|X_{u}^{N}(x)-X_{\tau_{u}}^{N}(x)\right|\right\|_{\mathbf{L}_{p}} \leq C_{p, \sqrt{31}} \frac{(1+|x|)}{N^{1 / 2}}, \\
\left\|\sup _{\tau_{t} \leq u \leq t}\left|X_{u}^{N}(x)-X_{u}^{N}(y)-X_{\tau_{u}}^{N}(x)+X_{\tau_{u}}^{N}(y)\right|\right\|_{\mathbf{L}_{p}} \leq C_{p, \sqrt{32}} \frac{|x-y|}{N^{1 / 2}} .
\end{array}
$$

Since $\max _{t \in[0, T]}\left|t-\tau_{t}\right|=\frac{T}{N}$, see $[27]$.

Proof. Here again, it is enough to prove the estimates for $p \geq 2$, which we assume from now on. Also we take $d=q=q^{\prime}=1$ to simplify the exposition. In order to have an accurate estimate, we do not use Corollary 2 as in the proof of Proposition 1, but Burkholder-Davis-Gundy's inequality combined with Jensen's inequality, and the fact that $\lambda(t) \leq \lambda_{\max }, \forall t \in[0, T]$. We obtain

$$
\begin{aligned}
\mathbb{E}\left(\sup _{\tau_{t} \leq u \leq t}\left|X_{u}^{N}(x)-X_{\tau_{u}}^{N}(x)\right|^{p}\right) & \leq 3^{p-1}\left\{\left(t-\tau_{t}\right)^{p-1} \int_{\tau_{t}}^{t} \mathbb{E}\left(\left|\mu\left(\tau_{s}, X_{\tau_{s}}^{N}(x)\right)\right|^{p}\right) \mathrm{d} s\right. \\
& +\left(t-\tau_{t}\right)^{p / 2-1}\left[C_{p}^{\mathrm{BDG}}\right]^{p}\left[\int_{\tau_{t}}^{t} \mathbb{E}\left(\left|\sigma\left(\tau_{s}, X_{\tau_{s}}^{N}(x)\right)\right|^{p}\right) \mathrm{d} s\right. \\
& \left.\left.+\lambda_{\max } \int_{\tau_{t}}^{t} \int_{\mathbb{R}} \mathbb{E}\left(\left|h\left(\tau_{s}, X_{\tau_{s}^{-}}, e\right)\right|^{p}\right) d s \nu(d e)\right]\right\} .
\end{aligned}
$$

According to Assumption (HP1), it follows that for any $t \in[0, T]$,

$$
\left\{\begin{array}{l}
|\mu(t, x)|+|\sigma(t, x)| \leq C^{X}(1+|x|) \\
|h(t, x, e)| \leq C^{X}(e)(1+|x|)
\end{array}\right.
$$

combined with 29), we deduce

$$
\begin{aligned}
\mathbb{E}\left(\sup _{\tau_{t} \leq u \leq t}\left|X_{u}^{N}(x)-X_{\tau_{u}}^{N}(x)\right|^{p}\right) & \leq 3^{p-1}\left(C^{X}\right)^{p}\left(\left(t-\tau_{t}\right)^{p} 2^{p-1}\left(1+C_{p,[29\}}^{p}(1+|x|)^{p}\right)\right. \\
& \left.+\left[C_{p}^{\mathrm{BDG}}\right]^{p}\left(t-\tau_{t}\right)^{p / 2} 2^{p-1}\left(1+\left[C_{p,[29}^{p}+\lambda_{\max } \int_{\mathbb{R}}\left[C^{X}(e)\right]^{p} \nu(\operatorname{de})\right](1+|x|)^{p}\right)\right)
\end{aligned}
$$

which readily leads to the announced estimate (31). 
Let us now turn to the second inequality: The same arguments combined with Assumption (HP1) and (30) lead to

$$
\begin{aligned}
& \mathbb{E}\left(\sup _{\tau_{t} \leq u \leq t}\left|X_{u}^{N}(x)-X_{u}^{N}(y)-X_{\tau_{u}}^{N}(x)+X_{\tau_{u}}^{N}(y)\right|^{p}\right) \\
& \leq 3^{p-1}\left\{\left(t-\tau_{t}\right)^{p-1} \int_{\tau_{t}}^{t} \mathbb{E}\left(\left|\mu\left(\tau_{s}, X_{\tau_{s}}^{N}(x)\right)-\mu\left(\tau_{s}, X_{\tau_{s}}^{N}(y)\right)\right|^{p}\right) \mathrm{d} s\right. \\
& +\left[C_{p}^{\mathrm{BDG}}\right]^{p}\left(t-\tau_{t}\right)^{p / 2-1}\left[\int_{\tau_{t}}^{t} \mathbb{E}\left(\left|\sigma\left(\tau_{s}, X_{\tau_{s}}^{N}(x)\right)-\sigma\left(\tau_{s}, X_{\tau_{s}}^{N}(y)\right)\right|^{p}\right) \mathrm{d} s\right. \\
& \left.\left.+\int_{\tau_{t}}^{t} \int_{\mathbb{R}} \mathbb{E}\left(\left|h\left(\tau_{s}, X_{\tau_{s}^{-}}, e\right)\right|^{p}\right) d s \nu(d e)\right]\right\} \\
& \leq 2^{p-1}\left(C^{X}\right)^{p} C_{p, \sqrt[30]{30}}^{p}\left(\left(t-\tau_{t}\right)^{p}+\left(\left[C_{p}^{\mathrm{BDG}}\right]^{p}+\lambda_{\max } \int_{\mathbb{R}}\left[C^{X}(e)\right]^{p} \nu(d e)\right)\left(t-\tau_{t}\right)^{p / 2}\right)|x-y|^{p},
\end{aligned}
$$

which completes the proof since $\left|t-\tau_{t}\right| \leq \frac{1}{N}, \forall t$.

\subsubsection{Strong convergence results:}

In order to derive a sharp convergence result, we must take into account the temporal regularity, Assumption (HP3), of the the coefficients $\mu, \sigma$ and $h$.

Theorem 6. Let Assumptions (HP1) and (HP3 hold and set $\beta=\min \left(\alpha, \frac{1}{2}\right)$. Then, for any $p>0$ there exists a generic constant $C_{p, 35}$ such that for any $x \in \mathbb{R}^{d}$

$$
\left\|\sup _{t \leq T}\left|X_{t}(x)-X_{t}^{N}(x)\right|\right\|_{\mathbf{L}_{p}} \leq C_{p, \sqrt{35}} \frac{(1+|x|)}{N^{\beta}} .
$$

Furthermore, for any $\gamma<\beta$, the random variables $\left(N^{\gamma} \sup _{t \leq T}\left|X_{t}-X_{t}^{N}\right|\right)_{N \geq 1}$ converge almost surely to 0 as $N$ tends to $+\infty$.

Proof. To simplify, as in the previous lemma, we take $d=q=q^{\prime}=1$. By definition, we have

$$
\begin{aligned}
X_{t}(x)-X_{t}^{N}(x) & =\int_{0}^{t}\left(\mu\left(s, X_{s}(x)\right)-\mu\left(\tau_{s}, X_{\tau_{s}}^{N}(x)\right)\right) \mathrm{d} s+\int_{0}^{t}\left(\sigma\left(s, X_{s}(x)\right)-\sigma\left(\tau_{s}, X_{\tau_{s}}^{N}(x)\right)\right) \mathrm{d} W_{s} \\
& +\int_{0}^{t} \int_{\mathbb{R}}\left(h\left(s, X_{s^{-}}(x), e\right)-h\left(\tau_{s}, X_{\tau_{s}^{-}}^{N}(x), e\right)\right) \tilde{N}(d s, d e) .
\end{aligned}
$$

Take the power $p$ and the expectation, and use Burkholder-Davis-Gundy's inequality combined with Jensen's inequality

$$
\begin{aligned}
\mathbb{E}\left(\sup _{t \leq T}\left|X_{t}(x)-X_{t}^{N}(x)\right|^{p}\right) & \leq 3^{p-1}\left\{T^{p-1} \int_{0}^{T} \mathbb{E}\left(\left|\mu\left(s, X_{s}(x)\right)-\mu\left(\tau_{s}, X_{\tau_{s}}^{N}(x)\right)\right|^{p}\right) \mathrm{d} s\right. \\
& +T^{p / 2-1}\left[C_{p}^{\mathrm{BDG}}\right]^{p}\left[\left.\int_{0}^{T} \mathbb{E}\left(\mid \sigma\left(s, X_{s}(x)\right)-\sigma\left(\tau_{s}, X_{\tau_{s}}^{N}(x)\right)\right)\right|^{p}\right) \mathrm{d} s \\
& \left.\left.+\lambda_{\max } \int_{0}^{T} \int_{\mathbb{R}} \mathbb{E}\left(\left|h\left(s, X_{s^{-}}(x), e\right)-h\left(\tau_{s}, X_{\tau_{s}^{-}}^{N}(x), e\right)\right|^{p}\right) d s \nu(d e)\right]\right\}
\end{aligned}
$$

From now, all terms will be treated in the same way. Taking the last and write that

$$
\left|h\left(s, X_{s^{-}}(x), e\right)-h\left(\tau_{s}, X_{\tau_{s}^{-}}^{N}(x), e\right)\right|^{p}
$$




$$
\begin{aligned}
& =\mid h\left(s, X_{s^{-}}(x), e\right)-h\left(s, X_{s^{-}}^{N}(x), e\right)+h\left(s, X_{s^{-}}^{N}(x), e\right)-h\left(\tau_{s}, X_{s^{-}}^{N}(x), e\right) \\
& +h\left(\tau_{s}, X_{s^{-}}^{N}(x), e\right)-\left.h\left(\tau_{s}, X_{\tau_{s}^{-}}^{N}(x), e\right)\right|^{p} \\
& \leq 3^{p-1}\left(\left|h\left(s, X_{s^{-}}(x), e\right)-h\left(s, X_{\tau_{s}^{-}}^{N}(x), e\right)\right|^{p}+\left|h\left(s, X_{s^{-}}^{N}(x), e\right)-h\left(\tau_{s}, X_{s^{-}}^{N}(x), e\right)\right|^{p}\right. \\
& \left.+\left|h\left(\tau_{s}, X_{s^{-}}^{N}(x), e\right)-h\left(\tau_{s}, X_{\tau_{s}^{-}}^{N}(x), e\right)\right|^{p}\right) .
\end{aligned}
$$

Thanks to (HP1), (HP3), 29) in Proposition 2 and (31) in Lemma 1, one can easily deduce

$$
\begin{aligned}
& \int_{0}^{T} \int_{\mathbb{R}} \mathbb{E}\left(\left|h\left(s, X_{s^{-}}(x), e\right)-h\left(\tau_{s}, X_{\tau_{s}^{-}}^{N}(x), e\right)\right|^{p}\right) d s \nu(d e) \\
& \leq 3^{p-1} \int_{\mathbb{R}}\left[C^{X}(e)\right]^{p} \nu(d e)\left[\int_{0}^{T}\left(\mathbb{E}\left(\left|X_{s}(x)-X_{s}^{N}(x)\right|^{p}\right)+\mathbb{E}\left(\left(1+\left|X_{\tau_{s}^{-}}^{N}(x)\right|\right)^{p}\right)\left(s-\tau_{s}\right)^{p \alpha}\right) d s\right. \\
& \left.+C_{p,[31]} \frac{(1+|x|)^{p}}{N^{p / 2}}\right] \\
& \leq 3^{p-1} \int_{\mathbb{R}}\left[C^{X}(e)\right]^{p} \nu(d e)\left[\int_{0}^{T}\left(\mathbb{E}\left(\left|X_{s}(x)-X_{s}^{N}(x)\right|^{p}\right)+C_{p,[29} \frac{(1+|x|)^{p}}{N^{p \alpha}}+C_{p,[31} \frac{(1+|x|)^{p}}{N^{p / 2}}\right]\right. \\
& \leq C_{p}\left[\int_{0}^{T}\left(\mathbb{E}\left(\left|X_{s}(x)-X_{s}^{N}(x)\right|^{p}\right)+\frac{1+|x|}{N^{p \beta}}\right] .\right.
\end{aligned}
$$

For some generic constant $C_{p}$.

By the same reasoning for $\mu$ and $\sigma$ we deduce the existence of a constant $C_{p}$ such that

$$
\mathbb{E}\left(\sup _{t \leq T}\left|X_{t}(x)-X_{t}^{N}(x)\right|^{p}\right) \leq C_{p}\left(\int_{0}^{T}\left(\mathbb{E}\left(\sup _{u \leq s}\left|X_{s}(x)-X_{s}^{N}(x)\right|^{p}\right) d s+\frac{(1+|x|)^{p}}{N^{p \alpha}}\right) .\right.
$$

The proof is then achieved by applying the Gronwall's lemma

This result will be useful in the proof of our main result Theorem 5 (see section 3.6.3), but unfortunately it is not sufficient at all. In view of Theorem 1 and its assumptions (in particular (H3)), one should have a sup over $|x| \leq \lambda$ inside the $\mathbf{L}_{p}$-norm. This is the purpose of the next derivations.

Strong convergence (new results) We now aim at obtaining uniform in space convergence results. Let us start with an easy result.

Proposition 3. Assume (HP1), (HP3) and let $\beta=\min \left(\alpha, \frac{1}{2}\right)$. For any $p>0$ and any $\rho \in[0, \beta]$, there exists a generic constant $C_{p, \rho, 36,}$ such that

$$
\left\|X_{t}(x)-X_{t}^{N}(x)-X_{t}(y)+X_{t}^{N}(y)\right\|_{\mathbf{L}_{p}} \leq C_{p, \rho, \sqrt{36}}(1+|x|+|y|)^{1-\rho / \beta} \frac{|x-y|^{\rho / \beta}}{N^{\beta-\rho}}
$$

for all $x, y \in \mathbb{R}^{d}$ and $t \in[0, T]$. Furthermore, for any $p>0$ and any $\rho>0$, there exists a generic

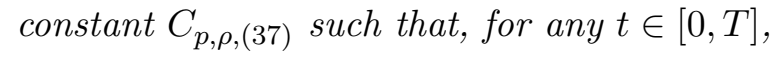

$$
\left\|\sup _{|x| \leq \lambda}\left|X_{t}(x)-X_{t}^{N}(x)\right|\right\|_{\mathbf{L}_{p}} \leq \frac{C_{p, \rho,(37)}}{N^{\beta-\rho}} \lambda, \quad \forall \lambda \geq 1 .
$$


The proof of this result is identical to that of the [GM18, Theorem 6].

As it is the case for Theorem 6, this result is not sufficient to derive Theorem 5. The next step is to generalize this two results. By making the best use of the regularity assumptions made on $\nabla_{x} \mu$ and $\nabla_{x} \sigma_{i}$ (see $(\mathbf{H P 2}$ ) and $(\mathbf{H P 4})$ ) we now obtain, in the following crucial Theorem, improved dependency in $N$ by allowing the case $\rho=0$.

Theorem 7. Let (HP1), (HP2), (HP3), (HP4) hold and let $\beta=\min \left(\alpha, \frac{1}{2}\right)$. For any $p>0$, there exists a generic constant $C_{p, 38,}$ such that

$$
\left\|\sup _{u \leq t}\left|X_{u}(x)-X_{u}^{N}(x)-X_{u}(y)+X_{u}^{N}(y)\right|\right\|_{\mathbf{L}_{p}} \leq C_{p, \sqrt{38} \mid}(1+|x|+|y|) \frac{|x-y|+|x-y|^{\delta}}{N^{\beta}}
$$

for all $x, y \in \mathbb{R}^{d}$ and $t \in[0, T]$. Furthermore, for any $p>0$ there exists a finite generic constant $C_{p, \sqrt{39})}$ such that, for any $t \in[0, T]$,

$$
\left\|\sup _{|x| \leq \lambda}\left|X_{t}(x)-X_{t}^{N}(x)\right|\right\|_{\mathbf{L}_{p}} \leq \frac{C_{p,(39)}}{N^{\beta}} \lambda^{2}, \quad \forall \lambda \geq 1 .
$$

Proof. As in the previous proofs, we argue that it is enough to assume $p \geq 2$. To alleviate the presentation, we additionally assume $d=q=q^{\prime}=1$, the derivation in the general case being similar. From the dynamics of $X$ and $X^{N}$, we write

$$
\begin{aligned}
& X_{t}(x)-X_{t}^{N}(x)-X_{t}(y)+X_{t}^{N}(y) \\
& =\int_{0}^{t}\left(\mu\left(s, X_{s}(x)\right)-\mu\left(\tau_{s}, X_{\tau_{s}}^{N}(x)\right)-\mu\left(s, X_{s}(y)\right)+\mu\left(\tau_{s}, X_{\tau_{s}}^{N}(y)\right)\right) \mathrm{d} s \\
& +\int_{0}^{t}\left(\sigma\left(s, X_{s}(x)\right)-\sigma\left(\tau_{s}, X_{\tau_{s}}^{N}(x)\right)-\sigma\left(s, X_{s}(y)\right)+\sigma\left(\tau_{s}, X_{\tau_{s}}^{N}(y)\right)\right) \mathrm{d} W_{s} \\
& +\int_{0}^{t} \int_{\mathbb{R}}\left(h\left(s, X_{s^{-}}(x), e\right)-h\left(\tau_{s}, X_{\tau_{s}^{-}}^{N}(x), e\right)-h\left(s, X_{s^{-}}(y), e\right)+h\left(\tau_{s}, X_{\tau_{s}^{-}}^{N}(y), e\right)\right) \tilde{N}(d s, d e)
\end{aligned}
$$

The same reasoning as in the proof of Proposition 1 leads to

$$
\begin{aligned}
& \mathbb{E}\left(\sup _{u \leq t}\left|X_{u}(x)-X_{u}^{N}(x)-X_{u}(y)+X_{u}^{N}(y)\right|^{p}\right) \\
& \leq 3^{p-1} t^{p-1} \int_{0}^{t} \mathbb{E}\left(\left|\mu\left(s, X_{s}(x)\right)-\mu\left(\tau_{s}, X_{\tau_{s}}^{N}(x)\right)-\mu\left(s, X_{s}(y)\right)+\mu\left(\tau_{s}, X_{\tau_{s}}^{N}(y)\right)\right|^{p}\right) \mathrm{d} s \\
& +3^{p-1}\left[C_{p}^{\mathrm{BDG}}\right]^{p} t^{p / 2-1}\{ \\
& \quad \times \int_{0}^{t} \mathbb{E}\left(\left|\sigma\left(s, X_{s}(x)\right)-\sigma\left(\tau_{s}, X_{\tau_{s}}^{N}(x)\right)-\sigma\left(s, X_{s}(y)\right)+\sigma\left(\tau_{s}, X_{\tau_{s}}^{N}(y)\right)\right|^{p}\right) \mathrm{d} s \\
& \left.\quad \times \lambda_{\max } \int_{0}^{t} \int_{\mathbb{R}} \mathbb{E}\left(\left|h\left(s, X_{s}(x)\right)-h\left(\tau_{s}, X_{\tau_{s}}^{N}(x)\right)-h\left(s, X_{s}(y)\right)+h\left(\tau_{s}, X_{\tau_{s}}^{N}(y)\right)\right|^{p}\right) \nu(d e) \mathrm{d} s .\right\}
\end{aligned}
$$

The three terms of the right side of above inequality can be treated in the same way, thus we only detail the computations for the last integral. First write that

$$
\begin{aligned}
& h\left(s, X_{s}(x), e\right)-h\left(\tau_{s}, X_{\tau_{s}}^{N}(x), e\right)-h\left(s, X_{s}(y), e\right)+h\left(\tau_{s}, X_{\tau_{s}}^{N}(y), e\right) \\
& =h\left(s, X_{s}(x), e\right)-h\left(s, X_{s}^{N}(x), e\right)-h\left(s, X_{s}(y), e\right)+h\left(s, X_{s}^{N}(y), e\right)
\end{aligned}
$$




$$
+h\left(s, X_{s}^{N}(x), e\right)-h\left(\tau_{s}, X_{\tau_{s}}^{N}(x), e\right)-h\left(s, X_{s}^{N}(y), e\right)+h\left(\tau_{s}, X_{\tau_{s}}^{N}(y), e\right) .
$$

Now, we treat the two lines above separately.

1. Denoting by $X_{s}^{N, \alpha, x}:=X_{s}(x)+\alpha\left(X_{s}^{N}(x)-X_{s}(x)\right)$ for $\lambda \in[0,1]$, we have

$$
\begin{aligned}
& h\left(s, X_{s}(x), e\right)-h\left(s, X_{s}^{N}(x), e\right)-h\left(s, X_{s}(y), e\right)+h\left(s, X_{s}^{N}(y), e\right) \\
& =\left(X_{s}(x)-X_{s}^{N}(x)-X_{s}(y)+X_{s}^{N}(y)\right) \int_{0}^{1} \nabla_{x} h\left(s, X_{s}^{N, \alpha, x}, e\right) d \alpha \\
& +\left(X_{s}(y)-X_{s}^{N}(y)\right) \int_{0}^{1}\left(\nabla_{x} h\left(s, X_{s}^{N, \alpha, x}, e\right)-\nabla_{x} h\left(s, X_{s}^{N, \alpha, y}, e\right)\right) d \alpha .
\end{aligned}
$$

By definition of $X^{N, \alpha, x}$, using the fact that $\left|\nabla_{x} h(t, x, e)\right| \leq C^{X, \nabla}(e)$ and $\left|\nabla_{x} h(t, x)-\nabla_{x} h(t, y)\right| \leq$ $C^{X, \nabla}(e)|x-y|^{\delta}$ with $\int_{\mathbb{R}}\left(C^{X, \nabla}(e)\right)^{p} \nu(d e)<\infty, \forall p \geq 2$; there exists a generic constant $C_{p}$ (whose values may change from line to line)

$$
\begin{aligned}
& \int_{\mathbb{R}}\left|h\left(s, X_{s}(x), e\right)-h\left(s, X_{s}^{N}(x), e\right)-h\left(s, X_{s}(y), e\right)+h\left(s, X_{s}^{N}(y), e\right)\right|^{p} \nu(d e) \\
& \leq C_{p}\left(\left|X_{s}(x)-X_{s}^{N}(x)-X_{s}(y)+X_{s}^{N}(y)\right|^{p}\right. \\
& \left.\quad+\left|X_{s}(y)-X_{s}^{N}(y)\right|^{p} \int_{0}^{1}\left|(1-\lambda)\left(X_{s}(x)-X_{s}(y)\right)+\lambda\left(X_{s}^{N}(x)-X_{s}^{N}(y)\right)\right|^{\delta p} \mathrm{~d} \lambda\right) \\
& \leq C_{p}\left[\left|X_{s}(x)-X_{s}^{N}(x)-X_{s}(y)+X_{s}^{N}(y)\right|^{p}\right. \\
& \left.\quad+\left|X_{s}(y)-X_{s}^{N}(y)\right|^{p}\left(\left|X_{s}(x)-X_{s}(y)\right|^{\delta p}+\left|X_{s}^{N}(x)-X_{s}^{N}(y)\right|^{\delta p}\right)\right]
\end{aligned}
$$

where we have used the Minkowsky inequality to handle the $d \lambda$-integral and also used the inequality

$$
(a+b)^{\gamma} \leq 2^{(\gamma-1)+}\left(a^{\gamma}+b^{\gamma}\right) \leq 2^{\gamma}\left(a^{\gamma}+b^{\gamma}\right), \forall a, b, \gamma \geq 0
$$

Now, we integrate over $(s, \omega)$ and apply the Cauchy-Schwarz inequality, to obtain

$$
\begin{aligned}
& \mathbb{E}\left[\int_{0}^{t} \int_{\mathbb{R}}\left|h\left(s, X_{s}(x), e\right)-h\left(s, X_{s}^{N}(x), e\right)-h\left(s, X_{s}(y), e\right)+h\left(s, X_{s}^{N}(y), e\right)\right|^{p} \nu(d e) \mathrm{d} s\right] \\
& \leq C_{p}\left[\int_{0}^{t} \mathbb{E}\left(\left|X_{s}(x)-X_{s}^{N}(x)-X_{s}(y)+X_{s}^{N}(y)\right|^{p}\right) \mathrm{d} s\right. \\
& \left.+\int_{0}^{t} \sqrt{\mathbb{E}\left(\left|X_{s}(y)-X_{s}^{N}(y)\right|^{2 p}\right)} \sqrt{\mathbb{E}\left(\left|X_{s}(x)-X_{s}(y)\right|^{2 \delta p}+\left|X_{s}^{N}(x)-X_{s}^{N}(y)\right|^{2 \delta p}\right)} \mathrm{d} s\right]
\end{aligned}
$$

which rewrites, owing to (14)-(30) and (35),

$$
\begin{aligned}
& \mathbb{E}\left[\int_{0}^{t} \int_{\mathbb{R}}\left|h\left(s, X_{s}(x), e\right)-h\left(s, X_{s}^{N}(x), e\right)-h\left(s, X_{s}(y), e\right)+h\left(s, X_{s}^{N}(y), e\right)\right|^{p} \nu(d e) \mathrm{d} s\right] \\
& \left.\leq C_{p}\left(\int_{0}^{t} \mathbb{E}\left(\mid X_{s}(x)\right)-X_{s}^{N}(x)-X_{s}(y)+\left.X_{s}^{N}(y)\right|^{p}\right) \mathrm{d} s+\frac{(1+|y|)^{p}}{N^{\beta p}}|x-y|^{\delta p}\right),
\end{aligned}
$$

for a new generic constant $C_{p}$.

2. Now we focus on the second line of identity (41). Similarly to before, we introduce for simplicity the notations

$$
\left\{\begin{array}{l}
\tilde{X}_{s}^{N, \alpha}(x):=X_{s}^{N}(x)+\alpha\left(X_{\tau_{s}}^{N}(x)-X_{s}^{N}(x)\right) \\
X_{\tau_{s}}^{N, \alpha}(x, y):=X_{\tau_{s}}^{N}(x)+\alpha\left(X_{\tau_{s}}^{N}(y)-X_{\tau_{s}}^{N}(x)\right)
\end{array}\right.
$$




$$
\begin{aligned}
& h\left(s, X_{s}^{N}(x), e\right)-h\left(\tau_{s}, X_{\tau_{s}}^{N}(x), e\right)-h\left(s, X_{s}^{N}(y), e\right)+h\left(\tau_{s}, X_{\tau_{s}}^{N}(y), e\right) \\
& =h\left(s, X_{s}^{N}(x), e\right)-h\left(s, X_{\tau_{s}}^{N}(x), e\right)-\left(h\left(s, X_{s}^{N}(y), e\right)-h\left(s, X_{\tau_{s}}^{N}(y), e\right)\right) \\
& +h\left(s, X_{\tau_{s}}^{N}(x), e\right)-h\left(s, X_{\tau_{s}}^{N}(y), e\right)-\left(h\left(\tau_{s}, X_{\tau_{s}}^{N}(x), e\right)-h\left(\tau_{s}, X_{\tau_{s}}^{N}(y), e\right)\right) \\
& =\int_{0}^{1} \nabla_{x} h\left(s, \tilde{X}_{s}^{N, \alpha}(x), e\right) d \alpha\left(X_{s}^{N}(x)-X_{\tau_{s}}^{N}(x)\right)-\int_{0}^{1} \nabla_{x} h\left(s, \tilde{X}_{s}^{N, \alpha}(y), e\right) d \alpha\left(X_{s}^{N}(y)-X_{\tau_{s}}^{N}(y)\right) \\
& +\int_{0}^{1} \nabla_{x} h\left(s, X_{\tau_{s}}^{N, \alpha}(x, y), e\right) d \alpha\left(X_{\tau_{s}}^{N}(x)-X_{\tau_{s}}^{N}(y)\right)-\int_{0}^{1} \nabla_{x} h\left(\tau_{s}, X_{\tau_{s}}^{N, \alpha}(x, y), e\right) d \alpha\left(X_{\tau_{s}}^{N}(x)-X_{\tau_{s}}^{N}(y)\right) \\
& =\int_{0}^{1}\left(\nabla_{x} h\left(s, \tilde{X}_{s}^{N, \alpha}(x), e\right)-\nabla_{x} h\left(s, \tilde{X}_{s}^{N, \alpha}(y), e\right)\right) d \alpha\left(X_{s}^{N}(x)-X_{\tau_{s}}^{N}(x)\right) \\
& +\int_{0}^{1} \nabla_{x} h\left(s, \tilde{X}_{s}^{N, \alpha}(y), e\right) d \alpha \times\left(X_{s}^{N}(x)-X_{\tau_{s}}^{N}(x)-X_{s}^{N}(y)+X_{\tau_{s}}^{N}(y)\right) \\
& +\int_{0}^{1}\left(\nabla_{x} h\left(s, X_{\tau_{s}}^{N, \alpha}(x, y), e\right)-\nabla_{x} h\left(\tau_{s}, X_{\tau_{s}}^{N, \alpha}(x, y), e\right)\right) d \alpha\left(X_{\tau_{s}}^{N}(x)-X_{\tau_{s}}^{N}(y)\right) .
\end{aligned}
$$

As in the first step of this proof, taking the power $p$ using the fact that $\left|\nabla_{x} h(t, x, e)\right| \leq C^{X, \nabla}(e)$ and $\left|\nabla_{x} h(t, x)-\nabla_{x} h(t, y)\right| \leq C^{X, \nabla}(e)|x-y|^{\delta}$ with $\int_{\mathbb{R}}\left(C^{X, \nabla}(e)\right)^{p} \nu(d e)<\infty, \forall p \geq 2$; there exists a generic constant $C_{p}$ such that

$$
\begin{aligned}
\int_{\mathbb{R}} \mid h(s, & \left.X_{s}^{N}(x), e\right)-h\left(\tau_{s}, X_{\tau_{s}}^{N}(x), e\right)-h\left(s, X_{s}^{N}(y), e\right)+\left.h\left(\tau_{s}, X_{\tau_{s}}^{N}(y), e\right)\right|^{p} \nu(d e) \\
\leq C^{X, \nabla} & {\left[\int_{0}^{1}\left|(1-\lambda)\left(X_{s}^{N}(x)-X_{s}^{N}(y)\right)+\lambda\left(X_{\tau_{s}}^{N}(x)-X_{\tau_{s}}^{N}(y)\right)\right|^{\delta} d \alpha\left|X_{s}^{N}(x)-X_{\tau_{s}}^{N}(x)\right|\right.} \\
& +\left|X_{s}^{N}(x)-X_{\tau_{s}}^{N}(x)-X_{s}^{N}(y)+X_{\tau_{s}}^{N}(y)\right| \\
& \left.+\left|s-\tau_{s}\right|^{\alpha} \int_{0}^{1}\left(1+\left|X_{\tau_{s}}^{N}(x)+\lambda\left(X_{\tau_{s}}^{N}(y)-X_{\tau_{s}}^{N}(x)\right)\right|\right) d \alpha\left|X_{\tau_{s}}^{N}(x)-X_{\tau_{s}}^{N}(y)\right|\right] .
\end{aligned}
$$

Integrating w.r.t. $(s, \omega)$, one can easily gets, for some new generic constant $C_{p}$

$$
\begin{aligned}
& \int_{0}^{t} \int_{\mathbb{R}} \mathbb{E}\left(\left|h\left(s, X_{s}^{N}(x), e\right)-h\left(\tau_{s}, X_{\tau_{s}}^{N}(x), e\right)-h\left(s, X_{s}^{N}(y), e\right)+h\left(\tau_{s}, X_{\tau_{s}}^{N}(y), e\right)\right|^{p}\right) \nu(d e) \mathrm{d} s \\
& \leq C_{p}\left[\int_{0}^{t}\left(\sqrt{\mathbb{E}\left(\left|X_{s}^{N}(x)-X_{s}^{N}(y)\right|^{2 p \delta}\right)}+\sqrt{\left.\left.\mathbb{E}\left(\mid X_{\tau_{s}}^{N}(x)-X_{\tau_{s}}^{N}(y)\right)\right|^{2 p \delta}\right)}\right)\right. \\
& \quad \times \sqrt{\mathbb{E}\left(\left|X_{s}^{N}(x)-X_{\tau_{s}}^{N}(x)\right|^{2 p}\right)} \mathrm{d} s \\
& \quad+\int_{0}^{t} \mathbb{E}\left(\left|X_{s}^{N}(x)-X_{\tau_{s}}^{N}(x)-X_{s}^{N}(y)+X_{\tau_{s}}^{N}(y)\right|^{p}\right) \mathrm{d} s \\
& \left.+\frac{1}{N^{\alpha p}} \int_{0}^{t}\left(1+\sqrt{\mathbb{E}\left(\left|X_{\tau_{s}}^{N}(x)\right|^{2 p}\right)}+\sqrt{\mathbb{E}\left(\left|X_{\tau_{s}}^{N}(y)\right|^{2 p}\right)}\right) \sqrt{\mathbb{E}\left(\left|X_{\tau_{s}}^{N}(x)-X_{\tau_{s}}^{N}(y)\right|^{2 p}\right)} \mathrm{d} s\right] .
\end{aligned}
$$

From results of Proposition 2 and Lemma 1, there is a new constant $C_{p}$ such that

$$
\begin{aligned}
& \mathbb{E}\left[\int_{0}^{t} \int_{\mathbb{R}}\left|h\left(s, X_{s}^{N}(x), e\right)-h\left(\tau_{s}, X_{\tau_{s}}^{N}(x), e\right)-h\left(s, X_{s}^{N}(y), e\right)+h\left(\tau_{s}, X_{\tau_{s}}^{N}(y), e\right)\right|^{p} \nu(d e) \mathrm{d} s\right] \\
& \leq C_{p}\left(\frac{|x-y|^{p \delta}}{N^{p / 2}}(1+|x|)^{p}+\frac{|x-y|^{p}}{N^{p / 2}}+\frac{|x-y|^{p}}{N^{\alpha p}}\left(1+|x|^{p}+|y|^{p}\right)\right) \\
& \leq C_{p}(1+|x|+|y|)^{p} \frac{|x-y|^{p}+|x-y|^{\delta p}}{N^{\beta p}}
\end{aligned}
$$


Thus, 411), 433 combined with (46) lead to

$$
\begin{aligned}
& \mathbb{E}\left[\int_{0}^{t} \int_{\mathbb{R}}\left|h\left(s, X_{s}^{N}(x), e\right)-h\left(\tau_{s}, X_{\tau_{s}}^{N}(x), e\right)-h\left(s, X_{s}^{N}(y), e\right)+h\left(\tau_{s}, X_{\tau_{s}}^{N}(y), e\right)\right|^{p} \nu(d e) \mathrm{d} s\right] \\
& \leq C_{p}\left[\int_{0}^{t} \mathbb{E}\left(\left|X_{s}(x)-X_{s}^{N}(x)-X_{s}(y)+X_{s}^{N}(y)\right|^{p}\right) \mathrm{d} s+(1+|x|+|y|)^{p} \frac{|x-y|^{p}+|x-y|^{\delta p}}{N^{\beta p}}\right],
\end{aligned}
$$

where $C_{p}$ is a new constant. The same estimates hold for $\mu$ and $\sigma$ instead of $h$. Hence, plugging the above into 40 , we obtain the existence of generic constants $C_{p}$ such that

$$
\begin{aligned}
& \mathbb{E}\left(\sup _{u \leq t}\left|X_{u}(x)-X_{u}^{N}(x)-X_{u}(y)+X_{u}^{N}(y)\right|^{p}\right) \\
& \leq C_{p}\left[\int_{0}^{t} \mathbb{E}\left(\sup _{u \leq s}\left|X_{u}(x)-X_{u}^{N}(x)-X_{u}(y)+X_{u}^{N}(y)\right|^{p}\right) \mathrm{d} s\right. \\
& \left.\quad+(1+|x|+|y|)^{p} \frac{|x-y|^{p}+|x-y|^{\delta p}}{N^{\beta p}}\right] \\
& \leq C_{p}(1+|x|+|y|)^{p} \frac{|x-y|^{p}+|x-y|^{\delta p}}{N^{\beta p}}
\end{aligned}
$$

where the last inequality follows from Gronwall's Lemma; the proof of $(38)$ is complete.

Let us now deduce (39) from (38) by applying Corollary 1 with $G(x):=X_{t}(x)-X_{t}^{N}(x)$. From (38) we have

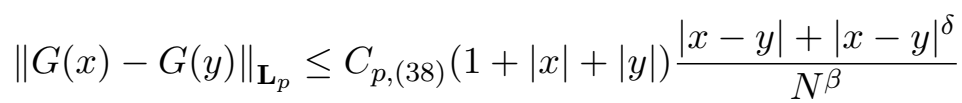

$$
\begin{aligned}
& \leq 2 C_{p, \sqrt{38}(1+|x|+|y|)^{2-\delta}} \frac{|x-y|^{\delta}}{N^{\beta}},
\end{aligned}
$$

using $|x-y|+|x-y|^{\delta}=|x-y|^{\delta}\left(1+|x-y|^{1-\delta}\right) \leq 2|x-y|^{\delta}(1+|x|+|y|)^{1-\delta}$. Thus, we can take $C^{(G)}=2 C_{p, \sqrt{38} /} / N^{\beta}, \tau^{(G)}=2-\delta$ and $\beta^{(G)}=\delta$ provided that $\delta \in(d / p, 1]$, which is true for $p$ large enough. We conclude as for the proof of (37).

We now have all the necessary elements to finalize the proof of our main result.

\subsubsection{Proof of Theorem 5}

We now carefully apply Theorem 1 with $F(\omega, x):=X_{t}(\omega, x), F^{N}(\omega, x):=X_{t}^{N}(\omega, x), \Theta:=$ $Y_{s}(\omega, y)$ and $\Theta^{N}:=Y_{s}^{N}(\omega, y)$.

(a) If the coefficients of $X$ and $Y$ satisfy (HP1) and (HP3).

1. As it is highlighted in Remark 3.1 Assumption (H1) is satisfied with $C_{p}^{\sqrt{\mathbf{H 1}}}:=C_{p, \text {,21 }}$ and $\alpha_{p}:=1$ (in view of Theorem 4 and $(\mathbf{H 2})$ is also satisfied for any given $\kappa \in(0,1)$ with $C_{p}^{\sqrt{\mathbf{H 2}}}:=C_{p, \sqrt{22}}$ (depending on $\kappa$ ) and $\alpha_{p}^{\sqrt{\mathbf{H 2} 2}}:=1-\kappa$.

2. (H3) is valid owing to Proposition 3 where, for any given $\rho>0$, we take $\varepsilon_{p}^{N, \overline{\mathbf{H 3} 3}}:=$ $\frac{C_{p, \rho, \sqrt{37}}}{N^{\beta^{X}-\rho}}\left(\right.$ with $\left.\beta^{X}:=\min \left(\alpha^{X}, \frac{1}{2}\right)\right)$ and $\alpha_{p}^{\sqrt{\mathbf{H 3}}}:=1$.

3. Finally, (H4) is clearly true using Propositions 1 and 2 applied to $Y$ instead of $X$,

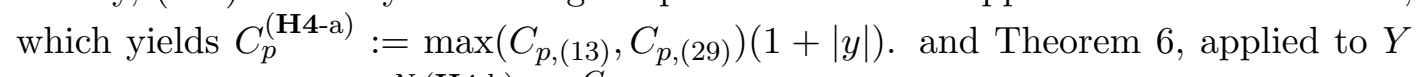
and $Y^{N}$, which gives $\varepsilon_{p}^{N, \text { H4-b }}:=\frac{C_{p, 35}}{N^{\beta^{Y}}}(1+|y|)$ with $\beta^{Y}:=\min \left(\alpha^{Y}, \frac{1}{2}\right)$. 
Thus, for any given $\kappa \in(0,1)$ and $\rho>0$, Theorem 1 gives

$$
\left\|X_{t}^{N}\left(Y_{s}^{N}\right)-X_{t}\left(Y_{s}\right)\right\|_{\mathbf{L}_{p}}=O\left(N^{-\kappa \beta^{Y}}+N^{-\beta^{X}+\rho}\right),
$$

thus the announced result.

(b) If in addition to the first case, the coefficients of $X$ satisfy $(\overline{\mathbf{H P 2}})$ and $(\overline{\mathbf{H P} 4})$. Assumption (H1) and (H4) are still valid. Assumption (H2) is satisfied with $\kappa=1$ thanks to (25). Assumptions (H3) holds as in the previous case, but now with $\rho=0$ owing to Theorem 7. The rest of the proof is unchanged, we are done.

\section{Approximation of utility-SPDE}

Our goal now is to apply the results of the previous section to the numerical approximation of the solution of the utility-SPDE (1). From (3), the marginal utility is the composition of two stochastic flows,

$$
U_{z}(t, z)=X_{t}\left(u_{z}\left(0, \xi_{t}(z)\right)\right), \quad U(0, z)=u(0, z)
$$

$\xi_{t}(z):=Y_{t}^{-1}(z)$ denotes the inverse flow of $y \mapsto Y_{t}(y)$. We assume that $X$ and $Y$ are two scalar SDEs with coefficients $(\mu, \sigma, h)$ and $(b, \gamma, g)$ respectively, driven by the same $q$-dimensional Brownian motion $B$ and the same Lévy measure (for simplicity we take $\lambda(t)=1$ for any $t$ ). Our aim is to give an approximation of $U_{z}$, using the results like those of Theorem 5 . For this, one last difficulty remains to be overcome: How to invert $Y$ ? We have identified four approaches for computing the inverse flow: (a) as an inverse of a random function; (b) as a forward in time SPDE; (c) as a forward in time SDE with stochastic coefficients; (d) as a backward in time SDE with standard coefficients.

In our paper [GM18, we have discussed these different approaches in detail (see [GM18, Section 4]) and we have exposed the main difficulties of each method. The simplest, most efficient and less expensive is the backward method. Without going into detail, this is the method we will adopt here.

Inverse flow as a backward in time SDE with standard coefficients. This approach asks to consider the dynamics of $\xi_{s, t}(x)$ in the variable $s$ : doing so, we aim at computing the inverse of $Y$ backward in time instead of forward in time. This approach relies on the following key result.

Theorem 8 ([Kun04, Theorems 3.11 and 3.13$])$. Suppose the coefficients $b, \gamma$ and $g$ of the SDE

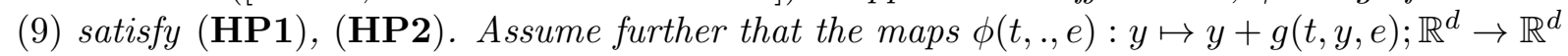
are homeomorphic with $I+\nabla g(t, y, e)$ is invertible for any $y$ a.e $(t, e)$.

(i) Then the solution $Y$ defines a stochastic flow of $\mathcal{C}^{1}$-diffeomorphisms.

(ii) If in addition the inverse maps $\psi(t, ., e)$ of $\phi(t, ., e)$ satisfy $\mathbf{H P 1}$ and $\gamma \in \mathcal{C}^{2,1}$ in $(t, x)$. Denote $k(t, z, e)=z-\psi(t, z, e)$ and assume that $\int_{\mathbb{R}}|k(t, z, e)-g(t, z, e)| \nu($ de $)$ is bounded. Then the inverse flow $\xi$ satisfies the following backward $S D E$

$$
\left\{\begin{aligned}
d \xi_{s, t}(z) & =\left[b\left(s, \xi_{s, t}(z)\right)-\partial_{x} \gamma\left(s, \xi_{s, t}(z)\right) \cdot \gamma\left(s, \xi_{s, t}(z)-\int_{\mathbb{R}} g\left(s, \xi_{s, t}(z), e\right) \nu(d e)\right] \hat{\mathrm{d}} s\right. \\
& +\gamma\left(t, \xi_{s, t}(z)\right) \cdot \mathrm{d} \overleftarrow{B}_{s}+\int_{\mathbb{R}} k\left(s, \xi_{s, t}(z), e\right) N(\hat{\mathrm{d} s}, d e) \\
\xi_{t, t}(z)= & z
\end{aligned}\right.
$$


The notations $\hat{\mathrm{d} s}$ and $\overleftarrow{B}_{s}$ are to remind that the integrals must be considered in a backward way. With this result in hand, the approximation of $\xi_{s, t}$ is made possible simply using a standard Euler scheme like for $X^{N}$. Using similar notations to those of Section 3.5. for $N \geq 1$, we consider the discretization family $\left\{\bar{t}_{i}:=i \frac{T}{N}, i \in \llbracket 0, N \rrbracket\right\}$ of $[0, T]$, the jump times $\left\{\theta_{k}, k \in \llbracket 1, J \rrbracket\right\}$ with corresponding marks $\left\{E_{k}, k \in \llbracket 1, J \rrbracket\right\}$ and finally the augmented partition

$$
\mathcal{P}_{N}:=\left\{t_{l}, l \in \llbracket 0, N+J \rrbracket\right\}:=\left\{\bar{t}_{i}:=i \frac{T}{N}, i \in \llbracket 0, \bar{N} \rrbracket\right\} \cup\left\{\theta_{k}, k \in \llbracket 1, J \rrbracket\right\}
$$

- Set $\xi_{t, t}^{N}(z)=z$. If $t \in\left\{\theta_{k}, k \in \llbracket 1, J \rrbracket\right\}$, set $\xi_{t^{-}, t}^{N}(z)=z-k\left(t, z, E_{t}\right)$ else $\xi_{t^{-}, t}^{N}(z)=z$.

- Let $t_{k_{N}}$ be defined as $\sup \left\{t_{k} \in \mathcal{P}_{N}: t_{k}<t\right\}$;

- For $s \in\left(t_{k_{N}}, t\right]$, set.

$$
\begin{aligned}
\xi_{s, t}^{N}(z) & =\xi_{t^{-}, t}^{N}-\gamma\left(t, \xi_{t^{-}, t}^{N}(z)\right) \cdot\left(B_{t}-B_{s}\right) \\
& -\left[b\left(t, \xi_{t^{-}, t}^{N}(z)\right)-\partial_{z} \gamma\left(t, \xi_{t^{-}, t}^{N}(z)\right) \cdot \gamma\left(t, \xi_{t^{-}, t}^{N}(z)\right)-\int_{\mathbb{R}} g\left(t, \xi_{t^{-}, t}^{N}(z), e\right) \nu(d e)\right](t-s) .
\end{aligned}
$$

- For $l \leq k_{N}$,

- if $t_{l} \in\left\{\theta_{k}, k \in \llbracket 1, J \rrbracket\right\}$ set $\xi_{t_{l}^{-}, t}^{N}(z)=\xi_{t_{l}, t}^{N}(z)-k\left(t_{l}, \xi_{t_{l}, t}^{N}(z), E_{t_{l}}\right)$ else $\xi_{t_{l}^{-}, t}^{N}(z)=\xi_{t_{l}, t}^{N}(z)$

- and for $s \in\left[t_{(l-1)}, t_{l}[\right.$

$$
\begin{aligned}
\xi_{s, t}^{N}(z) & =\xi_{t_{l}^{-}, t}^{N}(z)-\gamma\left(t_{l}, \xi_{t_{l}^{-}, t}^{N}(z)\right) \cdot\left(B_{t_{l}}-B_{s}\right) \\
& -\left[b\left(t_{l}, \xi_{t_{l}^{-}, t}^{N}(z)\right)-\partial_{x} \gamma\left(t_{l}, \xi_{t_{l}^{-}, t}^{N}(z)\right) \cdot \gamma\left(t_{l}, \xi_{t_{l}^{-}, t}^{N}(z)\right)-\int_{\mathbb{R}} g\left(t_{l}, \xi_{t_{l}^{-}, t}^{N}(z), e\right) \nu(d e)\right]\left(t_{l}-s\right) .
\end{aligned}
$$

Observe in (50) that as a difference with the usual Euler scheme, the first time step may be smaller than $T / N$ (instead of equal to): this choice has the slight advantage to simplify the overall numerical scheme since we use the same Brownian increments for both $X_{0, t}^{N}$ and $\xi_{0, t}^{N}$. Also, the fact that the two Euler schemes are built with the same Brownian motion (actually one is the time-reversal of the other) justifies the need for a general result like Theorems 1 and 5, available for arbitrary dependency in $F$ and $\Theta$.

Theorem 9. Assume the coefficients $(\mu, \sigma, h)$ of $X$ to satisfy $(\mathbf{H P 1})-(\mathbf{H P 2})-(\mathbf{H P 3})-(\mathbf{H P 4})$ (which $\alpha$-parameter is denoted by $\left.\alpha^{X}\right)$ and the coefficients $\left(b-\partial_{x} \gamma \cdot \gamma-\int g \nu(d e), \gamma, k\right)$ of $\xi_{., t}$ satisfy Assumptions (HP1)-(HP3) (which $\alpha$-parameter is denoted by $\left.\alpha^{Y}\right)$. Denote by $X_{0, .}^{N}$ the Euler approximation associated to $X_{0, .}$, with time step $T / N$, and by $\xi_{., t}^{N}$ the Euler approximation of the inverse flow $\xi_{., t}$ of $Y$, with time step $T / N$, according to (50)-(??).

Then, for any concave function $u$ with Lipschitz marginal utility $u_{z}$, the compound Euler scheme $X_{.}^{N}\left(u_{z}\left(\xi^{N}\right)\right)$ converges to $U_{z}(.,$.$) (solution to the SPDE of the form (1)) in any \mathbf{L}_{p}$-norm, at the order $\beta:=\min \left(\alpha^{X}, \alpha^{Y}, \frac{1}{2}\right)$ w.r.t. $N:$ For any $p>0$ and any $t \in[0, T]$,

$$
\left\|X_{0, t}^{N}\left(u_{z}\left(\xi_{0, t}^{N}(z)\right)\right)-U_{z}(t, z)\right\|_{\mathbf{L}_{p}}=O\left(N^{-\beta}\right) .
$$

The proof of this result is obvious. Without the function $u_{z}$, it would be a direct application of Theorem 5. However, since $u_{z}$ is Lipschitz, we easily check the estimates are unchanged.

Now having an accurate approximation of $U_{z}(t, z)$ using two Euler schemes (with computational cost equal to $2 N)$, we can easily retrieve $U(t, z)$ by standard numerical integration, using a known value, in general the utility of a zero wealth is equal to zero at any time, i.e., $U(t, 0)=0$. 
Conclusion: Numerical approximation of the solution of a stochastic PDE is not an easy task; it requires a lot of heavy computations because we have to discretize the solution and its derivatives using a finite differences method for example, which requires the resolution in the full space (or on a grid in $z$ ). This makes it extremely difficult to obtain strong convergence results. In this work as well as in GM18, we have shown that we can proceed otherwise in some situations where we can represent the solution as the compound of two random fields. In this case, the simplest scheme to approximate the SPDE's solution is the compound of the two schemes approximating $X$ and $Y$. The key point in our study is the regularity of $X$ with respect to its initial condition. It is this regularity, generally not considered too much in the literature, that allowed us to obtain the strong convergence rate for the compound and thus to avoid a discretization of the SPDE.

\section{References}

[AZ01] H. Allouba and W. Zheng. Brownian-time processes: the PDE connection and the half-derivative generator. Annals of probability, pages 1780-1795, 2001.

[BL93] N. Bouleau and D. Lépingle. Numerical Methods for Stochastic Processes. Wiley series in probability and mathematical statistics. Wiley \& Sons, Inc, 1993.

[BrPl07] N. Bruti-Liberati E. Platen Strong approximations of stochastic differential equations with jumps, Journal of Computational and Applied Mathematics, 205(2), pages 9821001,2007. Elsevier

[BY82] M.T. Barlow and M. Yor. Semi-martingale inequalities via the Garsia-RodemichRumsey lemma, and applications to local times. Journal of Functional Analysis, 49(2):198-229, 1982.

[EM13] N. El Karoui and M. Mrad. An exact connection between two solvable SDEs and a non linear Utility Stochastic PDEs. SIAM Journal on Financial Mathematics, 4(1):697$736,2013$.

[FuKun85] T. Fujiwara and H. Kunita and others. Stochastic differential equations of jump type and Lévy processes in diffeomorphisms group. Journal of mathematics of Kyoto University, 25(1), pages 71-106,1985. In Kyoto University.

[Gil08] M.B. Giles. Multilevel Monte Carlo path simulation. Operation Research, 56:607-617, 2008 .

[GM16] E. Gobet and M. Mrad. Strong approximation of stochastic processes at random times and application to their exact simulation. Stochastics, 10.1080/17442508.2016.1267179, 2016.

[GM18] E. Gobet and M. Mrad. Convergence rate of strong approximations of compound random maps, application to SPDEs. Discrete \& Continuous Dynamical Systems-B. 23 (10),2018. In American Institute of Mathematical Sciences.

[GRR70] A.M. Garsia, E. Rodemich, and H.Jr. Rumsey. A real variable lemma and the continuity of paths of some Gaussian processes. Indiana University Mathematics Journal, 20(6):565-578, 1970. 
[Hei01] S. Heinrich. Multilevel Monte Carlo Methods. In LSSC '01 Proceedings of the Third International Conference on Large-Scale Scientific Computing, volume 2179 of Lecture Notes in Computer Science, pages 58-67. Springer-Verlag, 2001.

[IkWa81] N. Ikeda and S. Watanabe. Stochastic Differential Equations and Diffusion Processes, In NewYork: North Holland Amsterdam, 1981.

[KS97] A. Kohatsu-Higa and M. Sanz-Solé. Existence and regularity of density for solutions to stochastic differential equations with boundary conditions. Stochastics Stochastics Rep., 60(1-2):1-22, 1997.

[KoTa10] A. Kohatsu-Higa and P. Tankov. Jump-adapted discretization schemes for Lévy-driven SDEs, Stochastic Processes and their Applications, 120(11), pages 2258-2285, 2010. Elsevier

[Kun97] H. Kunita. Stochastic flows and stochastic differential equations, volume 24 of Cambridge Studies in Advanced Mathematics. Cambridge University Press, 1997.

[Kun19] H. Kunita. Stochastic Flows and Jump-Diffusions, 2019. Springer.

[Kun04] H. Kunita. Stochastic differential equations based on Lévy processes and stochastic flows of diffeomorphisms, In Real and stochastic analysis, pages 305-373, 2004, Springer.

[MM20] A. Matoussi and M., Mrad. Dynamic Utility and related nonlinear SPDE driven by Lvy Noise. HAL, 2020.

[MS08] E. Mordecki, A. Szepessy, R. Tempone and G.E. Zouraris. Adaptive weak approximation of diffusions with jumps, in SIAM Journal on Numerical Analysis,46(4), pages1732-1768, 2008.

[MZ07] M. Musiela and T. Zariphopoulou. Investment and valuation under backward and forward dynamic exponential utilities in a stochastic factor model. In Advances in mathematical finance, pages 303-334. Birkhuser Boston, 2007.

[MZ10] M. Musiela and T. Zariphopoulou. Stochastic partial differential equations and portfolio choice. In Contemporary Quantitative Finance, pages 195-216. Springer, 2010.

[Nua06] D. Nualart. Malliavin calculus and related topics. Springer Verlag, second edition, 2006.

[OZ07] B. Øksendal and T. Zhang and others. The Itô-Ventzell formula and forward stochastic differential equations driven by Poisson random measures. Osaka Journal of Mathematics,44(1),pages 207-230, 2007. In Osaka University and Osaka City University, Departments of Mathematics.

[PrTa97] P. Protter and D. Talay. The Euler scheme for Lévy driven stochastic differential equations. The Annals of Probability, 25(1), 393-423, 1997.

[RG12] C. Rhee and P.W. Glynn. A new approach to unbiased estimation for SDEs. In C. Laroque, J. Himmelspach, R. Pasupathy, O. Rose, and A. M. Uhrmacher, editors, Proceedings of the 2012 Winter Simulation Conference, pages 495-503, 2012.

[RY99] D. Revuz and M. Yor. Continuous martingales and Brownian motion. Comprehensive Studies in Mathematics. Berlin: Springer, third edition, 1999. 